\title{
From social interactions to private environmental behaviours: The case of consumer food waste
}

\author{
Simone Piras ${ }^{\mathrm{a}}$, Simone Righi ${ }^{\mathrm{b}}$, Marco Setti ${ }^{\mathrm{c}}$, Nazli Koseoglu ${ }^{\mathrm{a}}$, Matthew J. Grainger ${ }^{\mathrm{d}}$, \\ Gavin B. Stewart ${ }^{\mathrm{e}}$, and Matteo Vittuaric

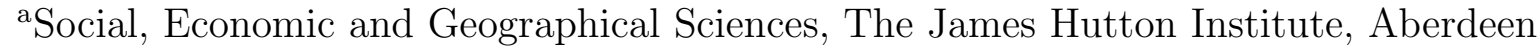 \\ AB15 8QH, Scotland, UK. Corresponding author. Email: simone.piras@hutton.ac.uk, \\ Tel: +44 (0)1224 395399 \\ ${ }^{b}$ Department of Economics, Ca' Foscari University of Venice, Fondamenta S. Giobbe 873, \\ 30121, Venezia, Italy. \\ ${ }^{\mathrm{c}}$ Department of Agricultural and Food Sciences, Alma Mater Studiorum - University of \\ Bolo-gna, Viale G. Fanin 50, 40137 Bologna, Italy. \\ ${ }^{\mathrm{d}}$ Department of Terrestrial Ecology, Norwegian Institute for Nature Research, NO-7485 \\ Trondheim, Norway.

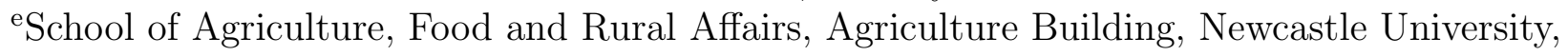 \\ Newcastle upon Tyne NE1 7RU, UK.
}

\begin{abstract}
Consumer food waste, like many environmental behaviours, takes place in private, and is not directly subject to social monitoring. Nevertheless, social interactions can affect private opinions and behaviours. This paper builds an agent-based model of interactions between consumers heterogeneous in their sociability, initial opinions and behaviours related to food waste and willingness to consider different opinions, in order to assess how social interactions can affect private behaviours. Compared to existing models of opinion dynamics, we innovate by including a range of "cognitive dissonance" between stated opinions and actual behaviours that consumers are willing to accept before changing one of the two. We calibrate the model using questionnaire data on household food waste in Italy. We find that a limited degree of mixing between different socio-demographic groups, namely adult and young consumers, is enough to trigger change, but a certain openness of mind is required from more wasteful individuals. Equally, a small group of environmentally committed consumers can attract a sizeable share of the population towards low-waste behaviours if they show a certain variability of opinions and are willing to compromise with individuals in their close neighbourhood in terms of opinions. These findings can help design effective interventions to promote pro-environmental behaviours, taking advantage of the beneficial network effects while anticipating negative externalities.
\end{abstract}

Keywords: Food waste; social interactions; consumer opinion; consumer behaviour; cognitive dissonance; agent-based model. 
This is the Accepted version of a paper with the same title that was published on October 10, 2021 in Resources, Conservation \& Recycling. The published version (and the supplementary material) is available at the address https://doi.org/10.1016/j.resconrec.2021.105952 and https://www.sciencedirect.com/science/article/pii/S0921344921005619.

\section{Introduction}

Food waste is a societal challenge drawing a burgeoning amount of attention in the agendas of public and private actors. In developed countries, consumers are responsible for the largest share of waste along value chains (Gustavsson et al. 2011; Gunders 2012; Stenmarck et al. 2016), equalling $53 \%$ of the total food waste in the EU. ${ }^{1}$ This implies the waste of valuable resources like soil, energy and water, but also the unnecessary emission of huge amounts of $\mathrm{CO}_{2}$ contributing to climate change. There has thus been an increasing focus on interventions to reduce food waste at the consumption stage. Most of the current literature on this matter focuses on the identification of socio-demographic and situational drivers (Wenlock et al. 1980; Sonesson et al. 2005; Wassermann and Schneider 2005; Barr 2007; WRAP 2014; Parizeau et al. 2015; Setti et al. 2016; Canali et al. 2017), and their relative influence (Grainger et al.2018b). A social component has also been identified (Evans 2011), suggesting that food waste is not simply an individual problem. However, there has been limited attention to the interaction between idiosyncratic and social aspects of consumers' food waste behaviour, including though simulation models. An attempt to study the process of consumers' food waste generation through extensive agent-based simulations has been made by Ravandi and Jovanovic (2019). However, their agent-based model (ABM) focuses on logistic issues in a very specific setting (food-service operations), with limited role for social influence.

To fill this gap, this paper merges evidence from the food waste literature on the one side, and social simulation on the other side, to develop an ABM that explores how individual food waste behaviours are influenced by peers through exchanges of opinions. In particular, we study the impact on food waste of interactions between individuals belonging to different socio-demographic groups. First, we focus on the role of mixing between adult and young consumers; second, we explore the convincing power of a small group of individuals committed to food waste reduction. We pay particular attention to the mismatch between publicly stated opinions and private behaviours. Unlike most ABMs, which are calibrated qualitatively using general stylized facts, our model is partially calibrated with real-world data.

Our simulations show that peers can influence each other's behaviour even when this behaviour is highly heterogeneous and private. Networks and similarity emerge as moderators between social influence and behavioural change. Rather than overall convergence, we observe the emerging of different clusters, as behaviours tend to align with individuals from the same group. The case study of adult and young consumers, with the former wasting little and the latter wasting much, suggests that if individuals are sufficiently open-minded, small degrees of mixing between groups are enough to reduce the share of those with "extreme" behaviours. The case study of populations with a small group of committed individuals shows that, under certain conditions, these can convince a significant share of the overall population. Being the first model to assess the impact of social influence on food waste generation though extensive simulations, there is a lack of research for

\footnotetext{
${ }^{1}$ Retrieved from: https://www.europarl.europa.eu/news/en/headlines/society/20170505ST073528/
} food-waste-the-problem-in-the-eu-in-numbers-infographic [Accessed 11.02.2021]. 
comparison. Nevertheless, our results suggest that policies addressing food waste should promote interaction opportunities among different socio-demographic groups to achieve larger impact.

The paper is structured as follows. First, we review the literature on food behaviours and social influence. Second, we describe our theoretical model and characterize our case studies starting from the literature and available data. Third, we present and discuss the results of extensive simulations. Finally, we draw our conclusions and illustrate their implications for research and policy design.

\section{Literature review}

Recent advancements in consumer studies have departed from the assumption that consumers make their decisions in isolation, based only on individual preferences and attitudes. The economics, psychology and sociology literature has been focusing on the impact of social embeddedness (i.e., of constraints due to the social context where economic activities take place) (Jackson 2005, 2010, Jackson et al. 2017), and peers' opinions (Galeotti 2010; Simpson et al. 2012; Wood and Hayes 2012; Lamberton et al. 2013; Carroni et al. 2020). When analysing the effects of social influence on behavioural change, social sciences identify two main moderators: existing networks (Abrahamse and Steg 2013), and perceived similarity with others (social comparison theory Festinger 1954). As for existing networks, Abelson et al. (1968) (quoted in Jackson 2005) show that individuals prefer to adopt behaviours coherent with their own and their peers' motivations. As for perceived similarity, a pattern coherent with the social comparison theory is observed when the reference group and the target group show similar characteristics (Goldstein et al. 2008), although this finding does not emerge in other parallel studies (Schultz et al. 2008). Moreover, when individuals identify strongly with a group, their behaviours tend to be consistent with norms of that group (social identity theory, Tajfel and Turner (2004)). The literature on social influence discusses the role of embeddedness by assessing how peers' opinions, including both "descriptive norms" (the consciousness of what others do) and "subjective/injunctive norms" (the perception about what others believe), map into individual motivations and actions (Ajzen 1991; Cialdini et al. 1990).

When referring to food, individual opinions and behaviours, and the influence that social norms exert on them, shows a unique complexity. The literature on consumer psychology has recognized that food choices result from a wide range of idiosyncratic factors, including moods, distraction, sensory cues, and psychology (Bublitz et al. 2010), as well as from social influence (McFerran et al. 2010). Food decisions tend to be driven by habits even when consumers report an intention to do otherwise (Ji and Wood 2007, Quested et al. 2013). Furthermore, people prefer to be in tune with others on the values and beliefs driving their food choices (Jackson 2005). In this regard, significant association has been found between subjective norms and individual opinions influencing food decisions (Graham-Rowe and Sparks 2015; Stancu et al. 2016).

Habits tend to play a role in consumers' food waste behaviour too (Lewin 1951; Verplanken et al. 1998; Stern 2000; Graham-Rowe et al. 2014). While ethnographic evidence has shown that food waste behaviour includes a social component (Evans 2011), the effect of habits tends to overcome personal intentions and to hinder social pressures even if consumers are averse to waste (Bolton and Alba 2012). Furthermore, food waste stems from private behaviours (Quested et al. 2013); therefore, it is not immediately visible to peers (Cecere et al. 2014) and no evidence of the relationship between descriptive norms and individual opinions emerge (Lapinski and Rimal 2005: Graham-Rowe and Sparks 2015). On the one hand, Lamberton et al. (2013) show that consumers' private decisions are grounded on one's own ideas and perceptions, and are thus less 
affected by others' reasoning in social interactions. On the other hand, Huh et al. (2014) found that consumers are more likely to mimic others' decisions when choosing in private, especially if their own preferences are not well-defined.

Why would individuals change their food waste behaviours as a result of opinion interactions with peers? Literature suggests that persuasion (Jackson 2005), social stigma (Kerr and Levine 2008; Kurzban and Leary 2001) and social punishment (Bowles and Gintis 2004; Dreber et al. 2008; Fehr and Gächter 2005; Fowler 2005; Fowler et al. 2005) can be effective in steering individual choices toward cooperative (socially optimal) outcomes. Consumer food waste behaviour at home is private; therefore, such strategies cannot work. Nevertheless, when choices are influenced by strong habits as in the case of food, people tend to adapt their behaviours to those of their peers through a process of mimetism to minimize their perceived distance from them (Van Baaren et al. 2004) and achieve consistency in their social relations (Jackson 2005). This process is not linear: in the context of food, attitudinal ambivalence, e.g. between enjoying large portions and avoiding waste, is common (Cornil et al. 2014). Piras et al. (2021) have shown that food waste is a social dilemma between care for the society (which is more common in areas with high social capital measured as political participation and blood and organ donations) and individual status deriving from food abundance. An "acceptable range of dishonesty" (i.e. discordance between behaviours and opinions) exists within which consumers do not experience personal discomfort (Argo et al. 2012), but if this range is overcome, they need to solve this contradiction by means of a cognitive mechanism (Festinger 1962).

The mismatch between publicly-stated opinions and private behaviours, also highlighted by the difference in food waste levels when detected through questionnaires and through waste sorting analysis (Giordano et al. 2018), represents a challenge for designing interventions aimed at tackling food waste. The literature has identified these dynamics at individual and social level, but to the best of our knowledge, their aggregate outcome in terms of behaviours has not been assessed. To explore how private food waste behaviours are affected by social exchanges of opinions in the presence of the above mismatch, we build an ABM based on the framework of opinion dynamics (Deffuant et al. 2002; Weisbuch et al. 2002; Hegselmann et al. 2002; Lorenz 2007; Galam 2008). In this class of models, individual opinions evolve through progressive coupling, leading to the creation of clusters of individuals with similar opinions. We draw further inspiration from Weisbuch et al. (2002) and Deffuant et al. (2002), who study populations where individuals have bounded confidence, i.e. are unwilling to compromise with individuals they consider too far from them. Namely, we include an opinion-distance threshold beyond which the agents are not updating their opinions in binary encounters. As recently shown by Young et al. (2017b), and debated by Young et al. (2017a) and Grainger and Stewart (2017), social media tools have some affect in reducing private food waste, but face-to-face interactions have a stronger potential to effectively modify behaviours. Hence, even if the interactions in our model could theoretically happen online, we developed it with face-to-face interactions in mind.

We extend the setup of Weisbuch et al. (2002) by allowing agents' opinions and behaviours to co-evolve, and the structure of social networks to constrain their interaction patterns. Individuals are modelled as heterogeneous in their sociability, their initial opinions and behaviours concerning food waste, their willingness to change opinion when interacting with peers (Nishi and Masuda 2013), and their tolerance for what we call "cognitive dissonance", i.e. the discordance between opinions and behaviours discussed above. For the sake of our analysis, simulated populations are divided into groups characterized by specific behavioural patterns. In each group, the variables are 
assigned either homogeneous values, or group-specific distributions. This modelling strategy allows to reduce unnecessary complexity (and thus complicatedness) by focusing on the group dychotomies which are of interest for the researcher, thus facilitating the identification of what variables drive the results. By means of extensive simulations, we study the impact on food waste behaviours of interactions between individuals belonging to different groups. After illustrating the baseline dynamic of the model by considering two theoretical groups, we calibrate it using empirical data and insights from the literature.

\section{Data and methods}

This section describes the theoretical model and the data used to characterize the two case studies through calibration.

\subsection{The theoretical model}

We consider a set of $\mathrm{N}$ agents $i \in\{1 \ldots, n\}$, representing single consumers (or, equivalently, households as unitary decision-maker: $\left.{ }^{2}\right)$. Each agent has a time-evolving food waste behaviour $W_{i}^{t} \in[0 ; 1]$ which measures the amount of waste generated in food routines (planning, purchasing, storing, preparing and cooking, serving and eating, disposing), with zero indicating the least wasteful behaviour. The behaviour of each agent is a private information, and is not observable by others. However, consumers exchange with their peers opinions on food waste $O_{i}^{t} \in[0 ; 1]$, e.g. on its acceptability or their concerns about its social and environmental impacts, with zero indicating opinions most averse to food waste.

Agents' interactions are bound by a network structure representing the ensemble of peers with which they exchange ideas and opinions (in line with Weisbuch et al. 2002). Indeed, social influence does not spread linearly among consumers but the position of individuals in their networks and their relative influenceability (here characterised as "openness of mind") matter (Watts and Dodds 2007). Formally, each agent $i$ has a set of peers $i^{\prime} \in \mathcal{S}_{i} \subset(N \neq i)$, with reciprocal connections, i.e. $i \in \mathcal{S}_{i^{\prime}} \Leftrightarrow i^{\prime} \in \mathcal{S}_{i}$. We consider the social network linking the individuals as an Erdös-Rényi random network topology (Erdös and Rényi 1959) with density $\lambda \in[0 ; 1]$. Random networks introduce a limited amount of variance in the network's degree, i.e. the number of connections of individual agents; however, as shown in Fig. 6in Appendix, our results are robust to the elimination of such variance. We refrain from adopting complex network structures, where the presence of abnormally connected individuals may complicate the interpretation of the results. Furthermore, despite characterizing the populations by attributing different social and waste features to different groups, we assume no ex-ante connection between agents' initial food waste and the characteristics of their networks. Finally, the model was developed with face-to-face interactions in mind but nothing prevents these dyadic interactions from happening online or via other communication tool. Instead, one-to-many communication (e.g., information diffused via TV or online platforms) is not considered in our setting but could be introduced as an additional treatment.

\footnotetext{
${ }^{2}$ In the real world, members of the same household can see and pass judgements based on each other's food waste behaviour, which cannot be observed publicly. However, to the best of our knowledge, consumers' food waste has only been measured at aggregated household level. Therefore, we treat food waste behaviours as pertaining to a single, unitary agent, and intra-household dynamics are out of the scope of our model.
} 
Interacting individuals tend to end up with opinions more similar to their peers. However, in line with Weisbuch et al. (2002), we assume our agents to be unwilling to compromise with agents expressing opinions which are too far from their priors. We define $d_{i n t}^{i} \in[0 ; 1]$ as the "interaction threshold", i.e. the distance beyond which others' opinions are not taken into consideration. It essentially measures the opposite of the intensity of the "confirmation bias" expressed by an individual. Interactions progress in discrete time steps, with one existing tie between two randomly-chosen agents $i$ and $i^{\prime}$ being selected at each step $t$. Then, the opinion of agent $i$ evolves according to the rule:

$$
O_{i}^{t+1}= \begin{cases}O_{i}^{t}+\mu\left(O_{i^{\prime}}^{t}-O_{i}^{t}\right) & \text { if }\left|O_{i^{\prime}}^{t}-O_{i}^{t}\right|<d_{i n t}^{i} \\ O_{i}^{t} & \text { otherwise }\end{cases}
$$

where $\mu \in[0 ; 0.5]$ is a parameter indicating the speed of convergence $(\mu=0.5$ in all simulations, meaning that a simple average between the opinions of the two agents is calculated; lower values would simply have the effect of slowing down the evolution of the model). The same applies to the interacting partner $i^{\prime}$. However, note that in Eq. 1, $d_{i n t}^{i}$ does not need to be equal to $d_{i n t}^{i^{\prime}}$. Thus, this setup admits interactions where consumer $i$ changes opinion while consumer $i^{\prime}$ does not, and vice versa, meaning social influence could be asymmetric. Like in the dyadic framework of decision-making proposed by Simpson et al. (2012), interacting consumers end up with intermediate opinions that are the result of reciprocal influence.

While agents' opinions start to evolve due to social interactions, waste behaviours (actions) do not necessarily evolve accordingly. Indeed, actions tend to be stickier than opinions. The fact that individual consumers declare a specific opinion when discussing with others (e.g., when reporting about their own food waste behaviour) while acting differently in private is a well-documented feature of food waste surveys (Quested et al. 2013; Moller et al. 2014a; Møller et al. 2014b; Setti et al. 2016; van Geffen et al. 2016: Giordano et al. 2018). Sengupta et al. (2002) find that misrepresentation is common in the context of social interactions among consumers when this is likely to generate a positive self-image, e.g. of an environmentally concerned person. This finding is especially relevant for food waste, whose avoidance carries strong "social desirability" (Giordano et al. 2018). However, if actions and opinions become too different compared to a given idiosyncratic threshold $d_{c d}^{i}$, i.e. when $\left|W_{i}^{t}-O_{i}^{t}\right|>d_{c d}^{i}$, agents start to suffer from cognitive dissonance, since they must defend in public an opinion they do not abide by in private. When this happens, the opinion $O_{i}^{t}$ and the action $W_{i}^{t}$ move closer. We define $I_{i}^{t}$ as the fraction of times an agent has modified their opinion (regardless of the size of the variation) over the number of past interactions, which is a proxy of their influenceability. If the number of past interaction is equal to zero, $I_{i}^{t}=0.5$. When agents start to suffer from cognitive dissonance, their action or opinion change according to this rule:

$$
\left\{\begin{array}{lll}
O_{i}^{t}=\left(1-I_{i}^{t}\right) O_{i}^{t}+I_{i}^{t} W_{i}^{t} & \text { with probability } & I_{i}^{t} \\
W_{i}^{t}=\left(1-I_{i}^{t}\right) O_{i}^{t}+I_{i}^{t} W_{i}^{t} & \text { with probability } & 1-I_{i}^{t}
\end{array}\right.
$$

This is in line with Mukhopadhyay et al. (2008) who enquired consumers' reaction to food temptations in the form of resisting or succumbing, finding that individuals end up repeating past decisions in the present ${ }^{3}$

Although social interactions tend to remain stable over time, when faced with an opinion that

\footnotetext{
${ }^{3}$ Let us note that, on the top of "cognitive dissonance", the society as a whole could be affected by a systematic "desirability bias" that applies to food waste actions. If this is the case, however, such bias would not qualitatively change the results of the model, but rather just imply a systematic shift (a bias) in these results.
} 
Table 1: Parameters and variables used in the model, symbols, and admitted values.

\begin{tabular}{|c|c|c|c|}
\hline Parameter & Symbol & Possible values & $\begin{array}{l}\text { Parameter val- } \\
\text { ues } \forall \text { simula- } \\
\text { tions }\end{array}$ \\
\hline Max number of time steps (dyadic encounters) & $t_{\max }$ & {$[1 ; \infty]$} & $4 \cdot 10^{5}$ \\
\hline Number of agents in the population & $N$ & {$[2 ; \infty]$} & 1,000 \\
\hline Food waste action of each agent $i$ at $t_{0}$ & $W_{i}^{0}$ & {$[0 ; 1]$} & \\
\hline Food waste opinion of each agent $i$ at $t_{0}$ & $O_{i}^{0}$ & {$[0 ; 1]$} & \\
\hline $\begin{array}{l}\text { Half-range of the distributions of } W_{i}^{0} \text { and } O_{i}^{0} \text { (for case } \\
\text { study calibration) }\end{array}$ & $\sigma$ & {$[0 ; 0.5]$} & \\
\hline Density of the network linking the agents & $\lambda$ & {$[0 ; 1]$} & 0.5 \\
\hline $\begin{array}{l}\text { Interaction threshold (distance beyond which others' } \\
\text { opinions are not taken into consideration) }\end{array}$ & $d_{\text {int }}^{i}$ & {$[0 ; 1]$} & \\
\hline $\begin{array}{l}\text { Speed of convergence of the opinions (weight in the } \\
\text { averaging mechanism) }\end{array}$ & $\mu$ & {$[0 ; 1]$} & 0.5 \\
\hline $\begin{array}{l}\text { Cognitive dissonance (max tolerated distance between } \\
W_{i} \text { and } O_{i} \text { ) }\end{array}$ & $d_{c d}^{i}$ & {$[0 ; 1]$} & \\
\hline $\begin{array}{l}\text { Weight in the movement of } W_{i} \text { towards } O_{i} \text { in case of } \\
\text { no previous interactions }\end{array}$ & $I_{i}^{0}$ & {$[0 ; 1]$} & 0.5 \\
\hline Distance $W_{i}^{t}-O_{i^{\prime}}^{t}$ over which a tie is eliminated & $d_{\text {act }}^{i}$ & {$[0 ; 1]$} & 0.1 \\
\hline Probability that a new tie is formed randomly & $P_{\text {rand }}$ & {$[0 ; 1]$} & 0.01 \\
\hline $\begin{array}{l}\text { Probability that an agent of group } j \text { is connected with } \\
\text { another individual from the same group } j\end{array}$ & $P_{\text {intra }}$ & {$[0 ; 1]$} & \\
\hline
\end{tabular}

is too distant from their private action, individuals could feel uncomfortable and thus reduce the contacts with the person expressing it. Formally, an agent $i$ considers whether $\left|W_{i}^{t}-O_{i^{\prime}}^{t}\right|<d_{a c t}^{i}$, and if this condition is not satisfied, they eliminate the tie and create a new one ${ }^{4}$ In line with the classical sociological literature (Simmel 1908), and specifically with the observation that most new ties are created with friends of friends (triadic closure), the new link is created with an individual already connected with one's friends. While this rule is followed in most cases, there is a small probability $P_{\text {rand }}<<1$ that the new link is formed randomly ${ }^{5}$

The parameters and variables in the model, their symbols, and their admitted range of values are illustrated in Table 1. For parameters, also the fixed values is thereby included. The model described in this section as well as all simulations are implemented in MATLAB 2016 6

\subsection{Model calibration}

The model described above is purely theoretical, i.e. no specific values are assigned to its parameters. To demonstrate the model's helpfulness in studying the evolution of food waste behaviours in a real population, we calibrate it using data on household food waste in Italy. We choose Italy because the model was developed at the University of Bologna, and because of the lack of evidence about the effectiveness of campaigns, and of large-scale simulations of food waste levels in this coun-

\footnotetext{
${ }^{4}$ In the simulations reported in this paper, $d_{a c t}^{i}=0.1 \forall i$; preliminary simulations showed that small variations around this value do not significantly change our results.

${ }^{5}$ In the simulations presented in this paper $P_{\text {rand }}=0.01$; preliminary simulations showed that small variations around this value do not change the outcome.

${ }^{6}$ All source codes used to create the figures and the data generated by the simulations are available at the following link: https://github.com/simonerighi/FoodWasteModel
} 
try. Italy represents an interesting case study due to the wide economic and socio-political diversity across its territory, which has been extensively studied in the literature (Putnam et al. 1993), and which results in different levels of social commitment towards environmental goals, including food waste reduction (Piras et al. 2021).

For calibrating the model, we use secondary data collected by the Italian National Observatory "Waste Watcher"'7 in 2015. "Waste Watcher" was established by Last Minute Market8 8 a spin-off of the University of Bologna that works towards food waste reduction through the recovery and redistribution of unsold food products. It has been running yearly surveys on consumer food waste since 2013. Although consumer food waste in Italy has been assessed through a number of onetime surveys (Gaiani et al. 2018), this is the longest available time series; this long-term experience allowed to progressively refine the questionnaire. The survey is administered via CAWI (ComputerAssisted Web Interviewing) by the market research company SWG.9 ${ }^{9}$ To achieve representativeness of the Italian population, quota sampling is adopted, with stratification by age, sex, income, and geographical area. Consumers belonging to a pool owned by the company are contacted via email, and asked to fill the questionnaire online until reaching the quotas. The 2015 survey involved a sample of 1,502 individuals aged 18 and above. Respondents had to be the responsible person, or one of the responsible persons, for food shopping, management or cooking within the household 10

In the "Waste Watcher" dataset, food waste behaviours are self-assessed and self-reported. This is done using three ordinal-scale questions on food waste frequency, quantity, and monetary value ${ }^{11}$ Despite slight differences, ${ }^{12}$ ordinal-scale questions are the norm in questionnaires to quantify food waste (Gaiani et al. 2018, Giordano et al. 2018). Giordano et al. (2018) found that self-assessed food waste levels are subject to underestimation. However, in most countries household food waste has been estimated exclusively through questionnaires due to the lower cost of this methodology, and this was the only available estimate of household food waste in Italy when we developed the model (Gaiani et al. 2018). Food waste opinions are detected by means of three ordinal-scale questions on the respondent's perception of the scale of the food waste problem, its seriousness, and how much they worry about it ${ }^{13}$ Although the main themes addressed by the survey remained the same along the years, specific questions, including those to assess food waste, have been refined based on new literature findings, projects, and empirical evidence, thus improving their effectiveness in detecting true population values.

Since opinions and behaviour of different individuals within a household could be heteroge-

\footnotetext{
${ }^{7}$ Waste Watcher: http://www.sprecozero.it/waste-watcher/ [Accessed 11.02.2021].

${ }^{8}$ Last Minute Market: http://www.lastminutemarket.it/[Accessed 11.02.2021].

${ }^{9}$ SWG: https://www.swg.it/[Accessed 11.02.2021].

${ }^{10}$ The questionnaire consisted of around 90 closed-ended questions (including conditional ones) on sociodemographic characteristics (about 30 questions), food routines, opinions on food waste or related issues such as the resources used to grow food, and policy interventions to prevent food waste. Two of the authors of this paper contributed to the development of some of the questions. Filling the questionnaire took 20-25 minutes on average. The dataset was provided to the authors by Last Minute Market. A subset of the questionnaire including all the questions used for calibration is provided as Supplementary Material. For research purposes, the full questionnaire can be requested to Last Minute Market.

${ }^{11}$ The questions and potential responses are provided as Supplementary Material. The frequency is measured on a five-point scale, the quantity on a six-point scale, and the value on a nine-point scale.

${ }^{12}$ For example, in 2013 the EU-level Eurobarometer survey asked about the percentage of food purchased that ended up as waste (Grainger et al. 2018a).

${ }^{13}$ The questions and potential responses are provided as Supplementary Material. The scale is measured on a three-point scale, the seriousness on a four-point scale, and the worrying on a four-point scale.
} 
neous, representing households with the response of a single member leads to reduced accuracy. Nevertheless, only a few studies have attempted to collect and analyse data on environmental behaviours from household members separately. For example, Longhi (2013) found that in the UK there are disparities in pro-environmental values and behaviour between household members when controlling for characteristics such as gender, age, education, and employment status. Seebauer and Wolf (2017) reached similar results after interviewing live-in couples in Austria. To the best of our knowledge, the individual vs household issue has never been investigated in the food waste literature where instead household size has been identified as one of the main determinants of waste levels (Grainger et al., 2018a b; Setti et al., 2016; Stancu et al., 2016; Parizeau et al., 2015; Secondi et al., 2015; Koivupuro et al. 2012; Barr, 2007). Indeed, due to the nature of the food waste phenomenon, individual waste flows are undistinguishable with any set of data, meaning that for all intent and purposes, the households need to be considered as unitary decision-makers. Also the "Waste Watcher" survey asks about food waste behaviours at the level of household but then measures the opinions of the individual respondent, implying that the latter is representative of the entire household and thus treating the household as a "black box". Equally, in our model the agents can be understood as households with a single aggregated food waste opinion and a single aggregated food waste behaviour, and whose links with other individuals are the sum of the links of all the household members. This assumption does not change the nature of the model, whose findings in terms of impact of social interactions remain valid. Entering intra-household dynamics leading to waste could be an interesting venue for future research, including modelling efforts.

While individuals are in principle all different from each other, we took steps to organize consumer differences around archetypal socio-demographic groups. Despite some degree of withingroup variation, it is reasonable to hypothesise intra-group heterogeneity to be smaller than intergroup heterogeneity. Therefore, we assume out the former, and focus on the impact of the latter. Some parameters, like the interaction thresholds $d_{i n t}^{i}$, are set as homogeneous within each group $j$ :

$$
\forall i \in j \quad d_{i n t}^{i}=d_{i n t}^{j} .
$$

Instead, we assume that the initial food waste actions $W_{0}$ and opinions $O_{0}$ of the individuals belonging to a group $j$ follow a distribution specific to that group. In particular, these variables are calibrated as follows. For each groups $j$, the probability mass function of the three behaviours and the three opinions is determined from the dataset; the $0-1$ range is divided into as many classes as the options in each question; for each agent $i$, and each of the three behaviours and the three opinions, a value in the $0-1$ range is extracted using the probability mass function; finally, the three behaviours and the three opinions are averaged to obtain respectively the initial food waste action $W_{0}^{i}$ and opinion $O_{0}^{i}$. The idiosyncratic "acceptable range of dishonesty" is set equal to the initial distance between the action and the opinion $d_{c d}^{i}=\left|W_{i}^{0}-O_{i}^{0}\right|$. This procedure implies a number of subjective choices. However, the purpose of this calibration is to set these key variables so that their distribution and correlation are qualitatively realistic rather than to achieve full representativeness of household food waste levels in Italy.

Differently from food waste, the values assigned to the parameters illustrating socio-psychological constructs $\left(\lambda, d_{\text {int }}^{i}, \mu, d_{c d}^{i}, I_{i}^{0}, d_{\text {act }}^{i}, P_{\text {rand }}\right.$ and $\left.P_{\text {intra }}\right)$ are purely theoretical. To the best of our knowledge, no studies in the food and food waste realm have ever tried to quantify them. For this reason, rather than exploring reality in its full complexity, for some of them we use a simple parametrisation described in the previous subsection. For others, we explore the impact on our 
output variable of changing their value endogenously through numerical comparative statics. This is a common practice in the ABM literature. If in the future these parameters will be measured by means of surveys, it will be possible to calibrate them too.

Identification of the case studies. As a first step, we identified homogeneous groups and their relative size within the sample. The dychotomy between young and adult people, with the former wasting more food, has been recognised as particularly relevant in the literature (Quested et al. 2013; Stefan et al. 2013, Watson and Meah 2013; WRAP 2014; Stancu et al. 2016). For this reason, our first case study focuses on this dychotomy, rather than on other ones such as education or income ${ }^{14}$ This case study is particularly relevant for Italy due to the strength of families and the importance of intergenerational contacts compared to other countries (e.g., the role of grandparents in children's education, Albertini 2016). Among others, this could favour the transmission of food management practices. For this first case study, survey respondents are classified as adults if aged 45 years or more ( $58 \%$ of the weighted sample), as young if aged between 18 and $44(42 \%)$. The choice of this threshold is a pragmatic one, aimed at generating two groups of similar size and deriving two conditional distributions; other thresholds could have been used without affecting the validity of the results.

Zemborain and Johar (2007) suggest that ambivalent individuals, who do not hold a clear opinion on an issue, are more open to persuasion. Therefore, a second case study is constructed around the dychotomy between committed and ordinary (non-committed) individuals. This is again a relevant case study for Italy, which has been characterised since its unification by a "silent majority" of moderate individuals (Gramsci 1975), and small elite groups extremely committed to universal value (thus, potentially, also environmentalism). Committed respondents are identified as those who selected the lowest available option in all the six questions on food waste behaviours and opinions. Overall, they account for $5.4 \%$ of the population in the "Waste watcher" sample. A Wilcoxon rank-sum test was used to assess if the variables of interest differ significantly across groups 15

Young vs adult consumers. In the "Waste watcher" sample, adults waste significantly less food both in terms of quantity and monetary value, and less frequently than the youth. After reporting these behaviours on a $0-1$ scale and averaging them as above, the mean resulting action is 0.18 for the youth and 0.12 for adults. Adult respondents are also significantly more concerned about food waste, more likely to think that the amount of food waste at societal level is large, and to deem it a serious problem for the planet. After applying the same transformation as above, the value of the opinion becomes 0.33 for adults and 0.37 for the youth. The average "range of dishonesty" is only slightly larger for adults, the difference being probably due to stronger habits; indeed, rather than being driven by an intention not to waste food, adults' behaviours are embedded in food activities, including planning and shopping routines (Stefan et al. 2013). Descriptive statistics are provided in Table 2.

In the simulations, the values of $W_{0}^{i}, O_{0}^{i}$ and $d_{c d}^{i}$ are assigned to each individual on the basis of data; instead, the interaction threshold $d_{i n t}^{i}$ is varied for young people to assess the sensitiveness

\footnotetext{
${ }^{14}$ Deriving more than two groups by cross-tabulating several variables would have resulted in small sub-samples, reducing representativeness and complicating the interpretability of the results in terms of mixing.

${ }^{15}$ This type of test is well-suited for ordinal data like most variables in our dataset, and is less sensible than a t-test to the presence of outliers in the skewed distributions of food waste opinions and behaviours.
} 
Table 2: Food waste indicators for young and adult respondents.

\begin{tabular}{lrrr}
\hline Variables & Youth & Adults & p-value (Wilcoxon) \\
\hline Share of households in the sample & 0.4185 & 0.5815 & - \\
\hline Food waste frequency (times per day) & 0.1212 & 0.0769 & 0.0000 \\
\hline Food waste quantity (kilograms per week) & 0.1895 & 0.1258 & 0.0000 \\
\hline Food waste value (Euros per week) & 7.24 & 5.14 & 0.0000 \\
\hline Food waste action (0=low; $1=$ high) & 0.1813 & 0.1235 & 0.0000 \\
\hline Perception of food waste scale $(1=$ big; $3=$ small) & 2.0675 & 1.9937 & 0.0012 \\
\hline $\begin{array}{l}\text { Seriousness of food waste for the planet (1=very seri- } \\
\text { ous; } 4=\text { not serious at all) }\end{array}$ & & & 0.0092 \\
\hline Worrying for food waste (1=a lot; 4=not at all) & 1.9770 & 1.6148 & 0.0223 \\
\hline Food waste opinion $(0=$ averse; $1=$ favourable) & 0.3665 & 0.3321 & 0.0000 \\
\hline $\begin{array}{l}\text { Cognitive dissonance (0=action equal to opinion; } \\
1=\text { maximum distance) }\end{array}$ & & & 0.2467 \\
\hline
\end{tabular}

Table 3: Parameters used for calibrating the "young vs adults" case study.

\begin{tabular}{llcl}
\hline Variables & Youth & Adults & Origin \\
\hline$t_{\text {max }}$ & \multicolumn{1}{c}{$4 \cdot 10^{5}$} & a priori \\
\hline$N$ & 418 & 582 & from data \\
\hline$W_{i}^{0}$ & 0.1813 & 0.1235 & from data \\
\hline$O_{i}^{0}$ & 0.3665 & 0.3321 & from data \\
\hline$\lambda$ & 0.5 & theoretical (see text) \\
\hline$d_{\text {int }}^{i}$ & {$[0.01 ; 0.16]^{*}$} & $0.06 \forall i \in A$ & tested endogenously \\
\hline$\mu$ & 0.5 & theoretical (see text) \\
\hline$d_{\text {cd }}^{i}$ & $\left|W_{i}^{0}-O_{i}^{0}\right|$ & from data \\
\hline$I_{i}^{0}$ & $0.5 \forall i$ & theoretical (see text) \\
\hline$d_{\text {act }}^{i}$ & $0.1 \forall i$ & theoretical (see text) \\
\hline$P_{\text {rand }}$ & 0.01 & theoretical (see text) \\
\hline$P_{\text {intra }}$ & {$[0 ; 1]^{* *}$} & tested endogenously \\
\hline
\end{tabular}

Notes: *in steps of $0.05 * *$ in steps of 0.025

of the model and thus of food waste to its change. Table 3 provides an overview of the calibration parameters used in this first case study.

Committed vs ordinary consumers. In the "Waste watcher" sample, committed individuals waste significantly less food in terms of both quantity and monetary value, and less frequently than ordinary individuals by definition. As a result, their average food waste action is zero, compared to 0.16 for ordinary people. Equally, committed respondents are more likely to be concerned about food waste, to think that the amount of food waste at societal level is large, and to consider it a serious problem for the planet. Their average food waste opinion is zero by definition, compared to 0.37 for ordinary people. The average "acceptable range of dishonesty" of committed individuals is zero by construction, but the difference with ordinary people is not statistically significant. Descriptive statistics are provided in Table 4.

Also for this second case study, in the simulations the values of $W_{0}^{i}, O_{0}^{i}$ and $d_{c d}^{i}$ are assigned to each agent on the basis of data, while the interaction threshold $d_{i n t}^{i}$ is varied for committed individuals to assess its impact on food waste levels. Even if the variability of $W_{0}^{C}$ and $O_{0}^{C}$ is zero by construction, to implement our sensitivity analysis we allow for some intra-group heterogeneity. 
Table 4: Food waste indicators for committed and ordinary respondents.

\begin{tabular}{lccl}
\hline Variables & Committed & Ordinary & $\begin{array}{l}\text { p-value } \\
\text { (Wilcoxon) }\end{array}$ \\
\hline Share of households in the sample & 0.0536 & 0.9464 & - \\
\hline Food waste frequency (times per day) & 0.0000 & 0.1008 & 0.0000 \\
\hline Food waste quantity (kilograms per week) & 0.0000 & 0.1611 & 0.0000 \\
\hline Food waste value (Euros per week) & 2.50 & 6.22 & 0.0000 \\
\hline Food waste action (0=low; $1=$ high) & 0.0000 & 0.1560 & 0.0000 \\
\hline Perception of food waste scale $(1=$ big; 3=small) & 1.0000 & 2.0826 & 0.0000 \\
\hline $\begin{array}{l}\text { Seriousness of food waste for the planet (1=very seri- } \\
\text { ous; } 4=\text { not serious at all) }\end{array}$ & 1.0000 & 1.6963 & 0.0000 \\
\hline Worrying for food waste (1=a lot; 4=not at all) & 1.0000 & 1.9752 & 0.0000 \\
\hline Food waste opinion $(0=$ averse; $1=$ favourable) & 0.0000 & 0.3662 & 0.0000 \\
\hline $\begin{array}{l}\text { Cognitive dissonance ( } 0=\text { action equal to opinion; } \\
1=\text { maximum distance) }\end{array}$ & 0.0000 & 0.2582 & 0.6206 \\
\hline
\end{tabular}

Table 5: Parameters used for calibrating the "committed vs ordinary" case study.

\begin{tabular}{|c|c|c|c|}
\hline Variables & Committed & Ordinary & Origin \\
\hline$t_{\max }$ & \multicolumn{2}{|c|}{$4 \cdot 10^{5}$} & a priori \\
\hline$N$ & 54 & 946 & from data \\
\hline$W_{i}^{0}$ & $\sigma^{C} \in[0 ; 0.2]^{*}$ & 0.1560 & tested endogenously; from data \\
\hline$O_{i}^{0}$ & $\sigma^{C} \in[0 ; 0.2]^{*}$ & 0.3662 & tested endogenously; from data \\
\hline$\lambda$ & \multicolumn{2}{|c|}{0.5} & theoretical (see text) \\
\hline$d_{i n t}^{i}$ & {$[0 ; 0.05]^{* *} \forall i \in C$} & $0.1 \forall i \in O r d$ & tested endogenously \\
\hline$\mu$ & \multicolumn{2}{|c|}{0.5} & theoretical (see text) \\
\hline$d_{c d}^{i}$ & \multicolumn{2}{|c|}{$\left|W_{i}^{0}-O_{i}^{0}\right|$} & from data \\
\hline$I_{i}^{0}$ & \multicolumn{2}{|c|}{$0.5 \forall i$} & theoretical (see text) \\
\hline$d_{a c t}^{i}$ & \multicolumn{2}{|c|}{$0.1 \forall i$} & theoretical (see text) \\
\hline$P_{\text {rand }}$ & \multicolumn{2}{|c|}{0.1} & theoretical (see text) \\
\hline$P_{\text {intra }}$ & \multicolumn{2}{|c|}{0.5} & theoretical (see text) \\
\hline
\end{tabular}

After assigning the values of $W_{0}^{i}$ and $O_{0}^{i}$ as described above, their values for committed agents are re-extracted from uniform distributions centred around $\overline{W_{0}^{C}}$ and $\overline{O_{0}^{C}}$ respectively, and with half-range $\sigma^{C} \in[0 ; 0.2]$. The impact of $\sigma^{C}$ is tested in the model. The values of $W_{0}^{i}$ and $O_{0}^{i}$ for ordinary individuals are kept as extracted initially. Table 5 provides an overview of the calibration parameters used in this second case study.

Interactions between groups. The members of a social group may (and in general are expected to) have different interaction patterns with peers from their group and individuals from other groups. Obtaining data on the actual patterns of interaction across groups is exceedingly difficult, but since the networks of the two pairs of groups considered in the simulations may not be fully mixed, we explore different degrees of intra- and inter-group influence. After dividing the population into two groups with their relative characteristic degrees, i.e. the average number of links of each agent $\lambda_{j}$ and $\lambda_{j^{\prime}}\left(\lambda_{j}=\lambda_{j^{\prime}}\right.$ in all subsequent simulations; indeed, as noted in Fig. 5 in Appendix, changing the relative level of $\lambda_{j}$ and $\lambda_{j^{\prime}}$ does not change the outcomes significantly), we model this feature by building the social networks according to the procedure hereby described. An individual 
belonging to group $j$ is connected with another individual from the same group $j$ with probability $P_{\text {intra }}$, and with individuals from the other group $j^{\prime}$ with probability $1-P_{\text {intra }}$. When $P_{\text {intra }}=0$, every individual is linked only with members of the other group; oppositely, when $P_{\text {intra }}=1$, the two groups are completely separated. A fully mixed network is obtained when $P_{\text {intra }}=0.5$.

\section{Results and Discussion}

As a preliminary step, we consider the dynamic behaviour of our model. Fig. 1 shows the results of baseline simulations where two equally-sized groups of agents (blue and red) are characterized by the same level of confirmation bias $d_{i n t}^{i}$, and the same level of cognitive dissonance $d_{c d}$. The network density $\lambda$ is equal to 0.5. In both groups, the initial levels of waste follow a triangular distribution, with $\operatorname{Tri}(\bar{W} \pm 0.33)$; however, the groups are heterogeneous in their initial average level of waste ( 0.33 for red consumers, 0.67 for blue ones). The left panel shows the evolution of the waste levels of each agent over time. Almost all the movements happen within the first 10,000 time-steps, especially among high-waste (blue) agents; then, the situation stabilizes, with clearly defined clusters emerging. Rather than converging towards an average level of waste, consumers tend to gather into four main equally-distanced clusters, and a smaller lower cluster; only a few individuals with very high waste remain isolated. Due to the perfect symmetry of our assumptions in this example all clusters are dominated by the type of consumers whose initial waste was closer to their centroid. However, the clusters located closer to the centre show a more diversified composition in terms of red and blue agents. By averaging the final waste of consumers across 100 simulations (central and right panels), it emerges that the final distribution of the agents in terms of waste depends on the relative openness of mind of the two groups. Allowing heterogeneity in the interaction threshold (i.e., if blue individuals, who waste more, are also more open-minded: right panel), the distribution becomes bi-modal, with a higher peak around low waste levels and a lower peak around mid-to-high waste levels. This happens because more open-minded (blue) individuals are more willing to compromise with others' opinions and thus tend to be convinced, and to move towards the position of less open-minded agents (red). The outcome changes sharply if the interaction threshold is the same for the two groups: the final distribution of waste resembles a bell-shaped distribution centered around 0.5, with a smaller peak around 0.7-0.8.

The emerging dynamics, showing multiple clusters rather than overall convergence of waste behaviours, finds a confirmation in reality but is not obvious from the modelling point of view. We replicate the findings of Weisbuch et al. (2002), whose model sees the emerging of multiple clusters "from an initial configuration where transitivity of opinion propagation [is] possible through the entire population: any two agents however different in opinions could [be] related through a chain of agents with closer opinions" (Weisbuch et al. 2002, p. 57). Such dynamics "end up in gathering opinions in clusters on the one hand, but also in separating the clusters in such a way that agents in different clusters do not exchange anymore" (Weisbuch et al., 2002, p. 57). If such situation materialises, further improvement in terms of food waste levels through opinion exchange becomes very difficult; therefore, "cognitive dissonance" could hinder the effectiveness of waste reduction efforts. Although a certain convergence is observed in the central clusters, in line with the social identity theory (Tajfel and Turner 2004), behaviours tend to align mostly with individuals from the same group, confirming that food waste has a social component (Evans $\mid 2011)$. 



Figure 1: Dynamic behaviour of the model. Left panel: temporal evolution of $W_{i}$ 's in a representative simulation. Central panel: distribution of $W_{i}^{t_{\max }}$ across 100 simulations (the interaction threshold is $d_{i n t}^{R}=0.10$ for red individuals and $d_{i n t}^{B}=0.20$ for blue individuals). Right panel: distribution of $W_{i}^{t_{\max }}$ across 100 simulations with interaction threshold $d_{\text {int }}^{B}=d_{\text {int }}^{R}=0.10$. Notes: In all panels $t_{\max }=4 \cdot 10^{5}, P_{\text {intra }}=0.5, d_{c d}=0.05, \overline{W^{R}}=0.33, \overline{W^{B}}=0.66$, and $\sigma=0.33$ for both groups. $N=1,000$ agents, equally divided between the two groups.

\subsection{Adults vs young consumers}

Having shown the dynamic evolution of the model and its dependency on the main parameters, we now assign them the values illustrated in Table 3 to simulate the young vs adults dychotomy, and focus on the stable states reached in the long run. This allows to understand how the two groups influence each other's opinions and actions, shading light on the conditions in which social processes drive the reduction of private anti-environmental behaviours. In line with our calibration, our populations include $58 \%$ of adult and $42 \%$ of young agents, and adults present lower waste levels $\left(\bar{W}_{0}^{A}=0.124\right)$ compared to young agents $\left(\overline{W_{0}^{A}}=0.181\right)$, which is also in line with the literature (Stancu et al. 2016). The "acceptable range of dishonesty" $d_{c d}^{i}$, and thus the likelihood that a change in opinion is translated into a change in behaviour, is set for each agent as equal to $\left|W_{i}^{0}-O_{i}^{0}\right|$, and does not differ significantly between the two groups. Since it is difficult to pinpoint the sign of the relationship between the confirmation bias of the two groups (which is largely dependent on individual and situational factors), we explore different cases by means of simulations. The interaction threshold for adults is thus fixed at $d_{i n t}^{A}=0.06$, while for young agents it varies between $d_{i n t}^{Y}=0.01$ and $d_{i n t}^{Y}=0.16$. This domain allows to explore situations where adults are both more and less open-minded than young consumers. Furthermore, as the groups of young and adults are unlikely to be fully mixed, we explore different patterns of intra- and inter-group mixing by changing $P_{\text {intra }}$. For each combination of $d_{i n t}^{Y}$ and $P_{\text {intra }}$, Fig. 2 shows the average and the standard deviation of the waste levels obtained with 100 simulations, while an analysis of the sensitivity of the variables plotted to the values of $d_{i n t}^{i}$ and $P_{\text {intra }}$ is provided as Supplementary Material. As noted in the baseline scenario, open-minded individuals tend to be attracted in the direction of less open-minded ones through progressive coupling. The analysis is focused on the relationship between the strength of this attraction and the strength of the interaction among groups.

Our sensitivity analysis shows that the relation between each of the variables plotted and the values of $d_{i n t}^{Y}$ and $P_{\text {intra }}$ is statistically significant regardless of the statistical model used to assess it. A key stylised fact emerging from Fig. 2 is that a large share of consumers tend to increase their waste levels, with more than $80 \%$ ending up with a waste above the third quartile of the initial 


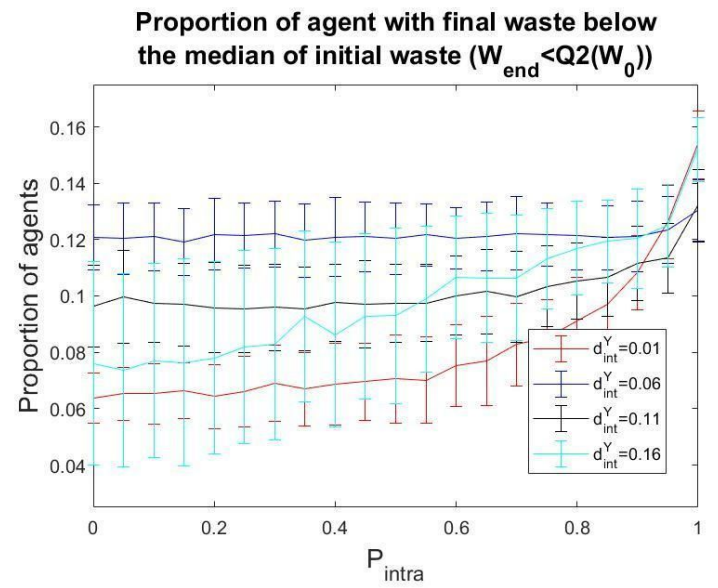

Proportion of agents with final waste below Q1

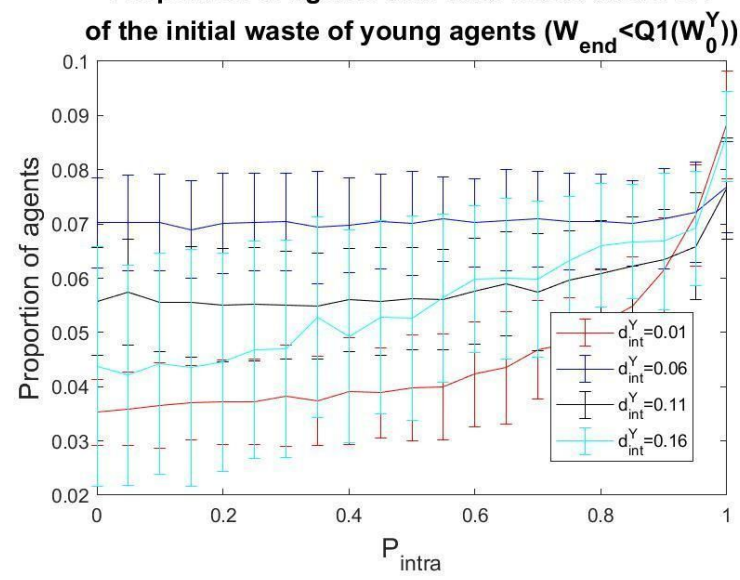

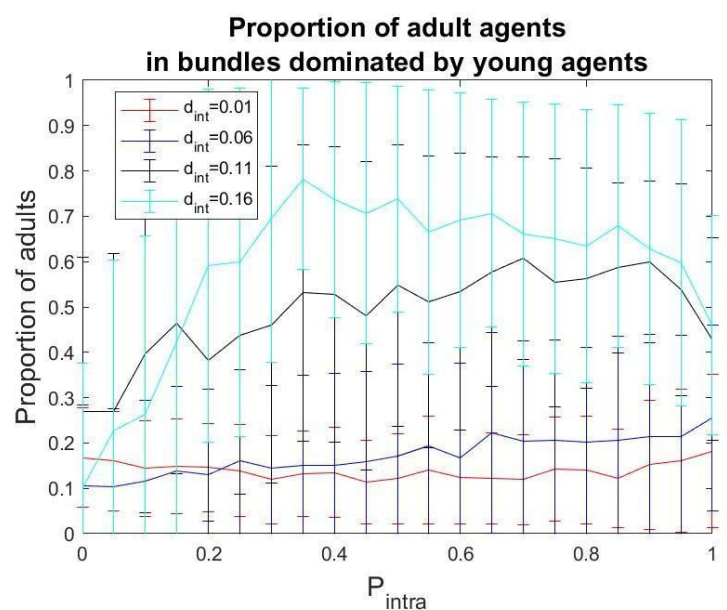

Proportion of agents with final waste above Q3 of the initial waste of adult agents $\left(W_{\text {end }}>Q 3\left(W_{0}^{A}\right)\right)$

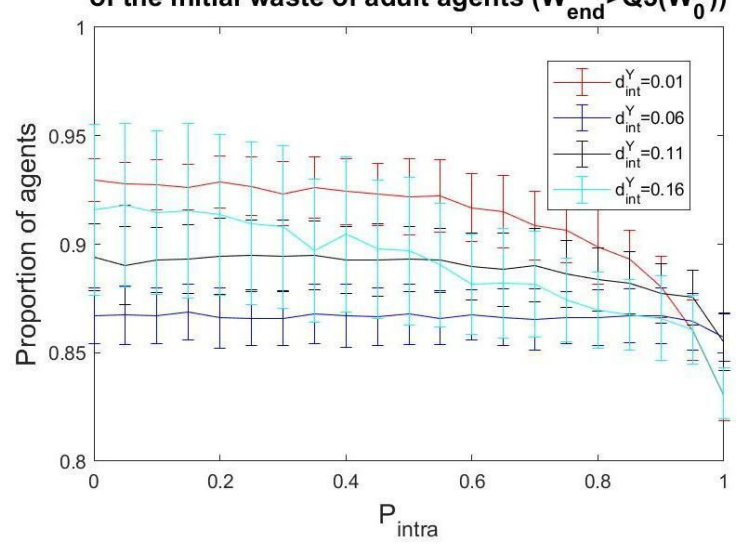

Figure 2: Adult vs young consumers. Top-left panel: proportion of agents with $W_{\text {end }}<$ median $\left(W_{0}\right)$. Top-right panel: proportion of adult agents ending up in bundles dominated (see definition in the text) by young agents. Bottom-left panel: proportion of agents with $W_{\text {end }}<$ $Q_{1}\left(W_{0}^{Y}\right)$. Bottom-right panel: proportion of agents with $W_{\text {end }}>Q_{3}\left(W_{0}^{A}\right)$. Notes: The calibration parameters are set in line with Table 3 . All the panels report the averages and standard deviations for changing levels of $P_{\text {intra }} \in[0 ; 1]$ across 100 simulations. The interaction threshold for adults is fixed at $d_{i n t}^{A}=0.06$, while we explore $d_{i n t}^{Y}=\{0.01,0.06,0.11,0.16\}$. 
waste of adult agents, and less than $15 \%$ with a waste below the initial median of the population. These large movements confirm that consumers can align their behaviour to what they think other people do (based on the latter's opinions) even when deciding in private (Huh et al. 2014). The increase in waste levels happens regardless of the initial setting, and is a result of calibration. Since in the "Waste watcher" dataset the opinions are much "higher" than the declared waste levels, they tend to attract the latter. A second relevant dynamic is represented by the interplay between the degree of mixing $P_{\text {intra }}$ and the interaction threshold $d_{i n t}^{i}$. If the interaction threshold differs between the two groups, small degrees of mixing $\left(0.8<P_{\text {intra }}<1\right.$, i.e. when less than $20 \%$ of the links are with agents from the other group) are enough to achieve sizeable movements, because less open-minded agents attract the members of the other group towards their position (in turn, the sensitivity analysis shows that values of $P_{\text {intra }}<0.25$ make a very small or non-significant difference compared to $P_{\text {intra }}=0$.). If the two groups present the same interaction threshold, the influence is reciprocal and the outcome in terms of waste levels does not depend on the level of mixing. Equally, increases in the level of mixing beyond $20 \%$ do not make a large difference for the final outcome for given levels of $d_{i n t}^{Y}$.

In case of no mixing between the groups, around $85 \%$ of the agents end up with a final waste above $Q_{3}\left(W_{0}^{A}\right)$ (bottom-right panel of Fig. 2), around $14 \%$ with a waste below median $\left(W_{0}\right)$ (topleft panel of Fig. 2), and only around $8 \%$ with a waste below the $Q_{1}\left(W_{0}^{Y}\right)$ (bottom-left panel of Fig. 2). In a fully mixed population $\left(P_{\text {intra }}=0.5\right)$, the share of agents with a waste above $Q_{3}\left(W_{0}^{A}\right)$ varies between about $86 \%$ and $93 \%$, while between $7 \%$ and $12 \%$ end up below median $\left(W_{0}\right)$, and between $3 \%$ and $7 \%$ below $Q_{1}\left(W_{0}^{Y}\right)$. Given the generalised increase in waste levels, rather than the absolute changes it is more interesting to discuss the changes comparatively for different parameter settings. The highest waste levels are observed when $d_{i n t}^{Y}<d_{i n t}^{A}$ and young agents are thus less willing to compromise with less wasteful ones (red lines). Interestingly, the lowest waste levels are not achieved when the interaction threshold of the young is much higher than the one of the adults $\left(d_{i n t}^{Y}=0.16\right.$, light blue lines), but when the two groups present similar interaction thresholds $\left(d_{i n t}^{Y}=0.06\right.$, blue lines $)$. This is because large interaction thresholds imply contrasting influences ending up in less movement, as confirmed by the standard deviation of the final waste levels, which is much larger when the agents present a large interaction threshold (light blue bars), especially for higher levels of mixing $\left(P_{\text {intra }}>0.5\right)$. Finally, the top-right panel of Fig. 2 suggests that young consumers are more likely to dominate a bundl ${ }^{16}$ when they present a larger interaction threshold and the degree of mixing is neither too low nor too high $\left(0.2<P_{\text {intra }}<0.8\right)$. The graph does not allow to determine the reason of this outcome. However, it is likely that young agents move towards adults until becoming a majority in many adults' groups, while adults make smaller movements towards the young (as shown by the increasing levels of waste). The relationship between the relative threshold of interaction and the "power of influence" is not monotonic: the share of adults in bundles dominated by young consumers (and the variability of this outcome) increases with increasing open-mindedness of the latter.

\footnotetext{
${ }^{16} \mathrm{~A}$ bundle is characterized as a set of consumers less distanced among themselves than $\max \left(P_{c d}, P_{\text {int }}\right)$, a radius equal to the maximum between the interaction threshold and the "cognitive dissonance" threshold. Such a group cannot further converge because the cognitive dissonance mechanism is never triggered and their interactions at the level of opinions do not result in a change in actions. This is similar to the mechanism that causes the emerging of non-converging clusters in Weisbuch et al. (2002). The dominance of a group of agents within a bundle is assessed by considering their relative share within that bundle. A bundle of consumers at $t_{\text {max }}$ is classified as dominated by agents of type $j$ if their proportion in the group is larger than their proportion in the population plus two standard deviations (computed relative to the size of the group).
} 
Overall, the statistical sensitivity analysis shows that, considering each agent separately across 8,400 simulations, the absolute change in the individual waste level is negatively related to the initial waste level $W_{0}^{i}$ (agents wasting more initially tend to move less), $d_{i n t}^{i}$ (more open-minded agents move less, probably because they are subject to more contrasting influences), and $P_{\text {intra }}$ (more links inside the group result in smaller movements). Instead, the change is positively related to the initial opinion $O_{0}^{i}$ (as expected, since in our calibration opinions are more favourable to waste than behaviours), and the cognitive dissonance $d_{c d}^{i}$ : agents willing to tolerate a larger mismatch between opinions and behaviours move more, which is partly counterintuitive and an interesting emerging dynamic of the model.

The stylised facts described in this subsection allow to draw useful recommendations. First, a small degree of mixing between socio-demographic groups (in this case young and adult consumers) is enough to activate reciprocal influence mechanisms. Virtuous (but potentially also wasteful) opinions spread more easily when "points of contact" are identified among reluctant people, and these are empowered of convincing their fellows, than in case of persistent and widespread campaigns. The benefits of a marginal increase in the degree of mixing beyond $P_{\text {intra }}=0.8$ (i.e., when young consumer have $20 \%$ of their links with adults, and vice versa) are not worth the likely increasing costs of promoting interaction opportunities. Second, if two socio-demographic groups have different degrees of open-mindedness, the outcome in terms of waste levels can be non-trivial, as less wasteful individuals can also be attracted towards less virtuous behaviours. While a certain open-mindedness of wasteful people is a necessary condition to achieve a reduction in waste levels, too high willingness to interact can have a counterproductive effect. At the limit, if two groups present the same interaction threshold, the average levels of waste in the population do not change significantly as a result of their encounter. Therefore, policymakers should target their campaigns carefully to avoid recoil effects.

\subsection{The role of committed individuals}

In real-world situations, populations made up of clear-cut groups are rarely observed. Rather, a homogeneous population may include small groups of individuals who campaign for specific issues, such as food waste reduction. Given the specific dynamics involving extreme opinions highlighted in the literature (Deffuant et al. 2002), it is crucial to explore the power of committed individuals in convincing their peers to converge toward their behaviour.

We now generate populations that, in line with the parameters in Table 5 , include $5.4 \%$ of committed agents. These take an "extreme" stance on food waste, which translates into low initial food waste actions and opinions. As described in the calibration section, the values of their $W_{0}^{i}$

and $O_{0}^{i}$ are extracted from uniform distributions centred around $\overline{W_{0}^{C}}$ and $\overline{O_{0}^{C}}$ respectively, and with half-range $\sigma^{C} \in[0 ; 0.2]$. Committed agents are also assumed to barely change their opinion, and only when engaging with individuals whose opinions are close to theirs. This translates into a small interaction threshold $\left(0<d_{i n t}^{C}<0.05\right)$. In turn, the initial food waste levels of ordinary agents are calibrated using the procedure described in the Model Calibration subsection, and their open-mindedness is set at $d_{i n t}^{\text {Ord }}=0.1$. Again, for each agent the threshold of "cognitive dissonance" is equal to $\left|W_{i}^{0}-O_{i}^{0}\right|$, and thus smaller for committed agents by construction. Finally, the network is supposed to be fully mixed $\left(P_{\text {intra }}=0.5\right)$, meaning that each agent has an equal probability to be connected with agents from either group. 
To test the power of attraction of committed individuals, we assess the proportion of ordinary consumers ending up in bundles dominated by the former and the overall proportion of agents ending up with waste levels below the initial median of the population, both at $t_{\max }$. We implement a statistical analysis by progressively changing the interaction threshold of committed individuals $d_{i n t}^{C}$ from 0 to 0.05 in steps of 0.0025 , and the half-range of their initial uniformly distributed food waste opinions and behaviours $\left(\sigma^{C}\right)$ from 0 to 0.2 in steps of $0.0025{ }^{17}$ The results for each possible combination of the two parameters are illustrated in Fig. 3, while a statistical analysis is provided as Supplementary Material.
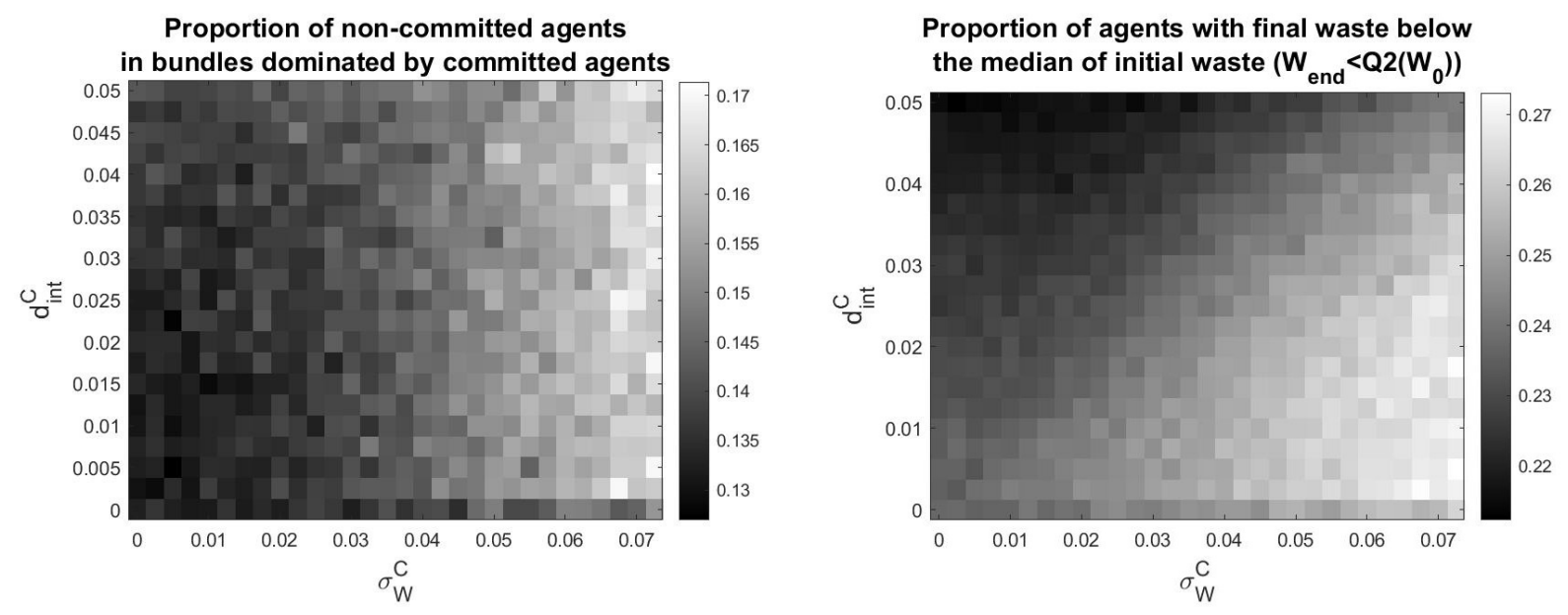

Figure 3: Committed vs ordinary consumers. Left panel: Proportion of ordinary agents ending up in bundles dominated (see definition in the text) by committed agents. Right panel: Proportion of agents with $W_{\text {end }}<$ median $\left(W_{0}\right)$. Notes: The calibration parameters are set in line with Table 5. The colours of each cell are based on averages across 100 simulations for all possible combinations of $d_{i n t}^{C} \in[0 ; 0.05]$ (in steps of 0.0025) and $\sigma^{C} \in[0 ; 0.2]$ (in steps of 0.0025).

Like in the previous case study, to align with the calibration dataset, the opinions are initialised as more "favourable" to food waste compared to the behaviours. Furthermore, since the "range of dishonesty" that committed individuals are willing to accept is narrower on average, their behaviours are more likely to align with their opinions. Given these premises, the final waste levels are much higher than the initial ones: the proportion of agents ending up with $W_{\text {end }}<$ median $\left(W_{0}\right)$ ranges between 22-27\% depending on the setting (right panel of Fig. 3). This is still a better outcome than in the previous case study, where between $6-16 \%$ of the agents ended up below median $\left(W_{0}\right)$ (upper-left panel of Fig. 2), pointing to the beneficial impact of committed groups when specific conditions - detailed below - are verified. Another key stylised fact emerging from Fig. 3 is that the combination of open-mindedness and variability of opinions matters more than the value of these parameters separately. The only exception is when $d_{i n t}^{C}=0$ (i.e., when committed agents do not engage with anyone, illustrated in the bottom line of the two panels): in this case the value of $\sigma^{C}$ does not matter. Committed people are thus doomed to have no relevance beyond their own group if they are not willing to compromise with the rest of the population. The three-way relationship between the openness of mind $d_{i n t}^{C}$, the variability of food waste $\sigma^{C}$, and

\footnotetext{
${ }^{17}$ Since $\overline{W_{0}^{C}}<0.2$ for all population, the actual range of $\sigma^{C}$ is smaller than 0.2 .
} 
final waste $W_{\text {end }}$ is not linear. The "best" outcomes are achieved when the openness of mind is relatively low $\left(d_{i n t}^{C}<0.03\right)$, and the variability of food waste for committed individuals relatively high $\left(\sigma^{C}>0.04\right)$. When committed agents are more spread, they are more likely to attract ordinary agents located within their interaction threshold towards their positions; however, if their "openness of mind" is too large, they may be attracted towards higher food waste levels in turn 18 The statistical sensitivity analysis reported in Supplementary Material confirms this: beside the significant coefficients linking the proportion of agents with $W_{\text {end }}<\operatorname{median}\left(W_{0}\right)$ to $d_{\text {int }}^{C}$ (negative) and $\sigma^{C}$ (positive), the interaction between the two parameters also yields a significant coefficient (negative). Compared to zero as a baseline, increasing values of $d_{i n t}^{C}$ yield first positive and then increasing negative results, while the impact of $\sigma^{C}$ is positive and increasing.

A similarly complex pattern is shown in the left panel of Fig. 3, although $d_{\text {int }}^{C}$ matters less in this case. The proportion of ordinary agents ending up in bundles dominated by committed agents is larger when the variability of the latter's food waste is relatively high $\left(\sigma^{C}>0.04\right)$, while for low variability relatively high levels of the openness of mind $\left(d_{\text {int }}^{C}>0.035\right)$ generate slightly better outcomes. The statistical sensitivity analysis confirms these observations, as both parameters are significantly and positively related to the variable plotted in the left panel of Fig. 3 , but their interaction term is non-significant. Equally, any value of $d_{\text {int }}^{C}>0$ yields a positive power of attraction of committed agents compared to $d_{i n t}^{C}=0$, but with no clear pattern, while increasing $\sigma^{C}$ yields a clearly increasing power of attraction. It is worth remembering that a bundle is dominated by committed agents when their proportion is larger than their proportion in the overall population plus two standard deviations. This proportion is $5.4 \%$; hence, these bundles can include a large majority of ordinary agents but are still subject to the influence of committed individuals. Indeed, most settings with a large proportion of ordinary agents ending up in bundles dominated by their counterpart are also characterised by lower final levels of waste (right panel of Fig. 3).

These results suggest that under certain conditions - such as sufficient within-group variation $\sigma^{C}$ and positive but not-too-large openness of mind $d_{\text {int }}^{C}$ - committed consumers can attract (or retain) a considerable share of individuals close to their ideas. We thus reproduce a result similar to Deffuant et al. (2002), whose model adopts relative agreement between pairs of individuals to simulate the emerging and the influence of extremism, but translate and embed it to the problem of food waste. To maximize their effectiveness in spreading virtuous food waste behaviours, committed individuals should allow a certain variability of opinions within their group and, at the same time, be willing to engage with people whose opinions are different from theirs but not too much. This result aligns well with Watts and Dodds (2007), which found that the presence of critical masses of easily influenceable individuals is more important than the presence of influencers in generating a cascade effect. It is also worth mentioning that more variability among committed individuals implies slightly higher food waste, suggesting that there is a trade-off between power of persuasion and the achievable unitary improvements.

Being the first model to study the impact of social interactions on food waste generation though extensive simulations, there is a lack of studies for comparison. More in general, our results suggest that, in line with Huh et al. (2014), social embeddedness and peers' choices can influence behaviour even when the latter shows a high heterogeneity and is private (i.e., takes place at home) like consumer food waste. In line with the literature on resource conservation, networks and similarity emerge as moderators between social influence and behavioural change (Abrahamse and Steg 2013),

\footnotetext{
${ }^{18}$ The results in Fig. 4 confirm these finding, being either symmetrical (left panel) or analogous (right panel) to the right panel of Fig. 3 .
} 
whereas in line with the social psychology theory, also in the food waste domain habits exert a sticky influence, i.e. resist change (Verplanken et al. 1998). Interaction opportunities among different socio-demographic groups should thus be considered by policies tackling food waste.

\section{Conclusions and policy implications}

The present model, in its simplicity, has uncovered interesting stylized facts concerning the influence of social interactions on private environmental behaviours, providing insights into behaviour change critical for the design of effective policy interventions. To this end, we have explored the effects of social interactions on stated opinions and private food waste behaviours by means of an ABM. We built two numerical case studies of heterogeneous populations by calibrating the model with Italian data on household food waste. First, we assumed our populations to be made of adult and young consumers, with the former wasting less on average than the latter, and observed the impact on food waste of their mixing. Then, we assessed the convincing power of a small group of committed individuals. Indeed, in a population divided into fairly homogeneous groups, policy interventions may choose to target specific groups, and inter-group interactions are likely to scale up their impact.

Our simulations show that even a low degree of mixing (i.e., a small number of inter-group interactions) is sufficient to convince individuals across groups, particularly those showing "extreme" behaviours to converge towards more "central" positions. Considering that the degree of mixing between adult and young consumers is higher where interactions between different generations are more frequent, these results suggest that extended households and houses of multiple occupancy could be characterized by a smaller number of individuals showing "extreme" food waste behaviours. While we calibrated our model using real food waste data, the network linking the agents is an Erdös-Rényi random network with density $\lambda=0.5$ - a value which is not based on a real population. In Italy, the interactions between people of different age are more common than in other EU counties (Albertini 2016), suggesting that the patterns of behaviour diffusion can be location-specific, and our findings do not necessarily extend to all countries when local network structures are factored in.

Additionally, we found that a small number of committed consumers can effectively attract (or retain) other consumers close to their position if they show some within-group variability of opinion and are willing to consider different opinions but not too different. Open-mindedness alone does not allow them to reach out to a relevant number of individuals, while variability alone is not enough to ensure that they engage with ordinary people without being attracted towards them instead. The optimum in terms of convincing power is achieved when the opinions of committed individuals are spread across a certain range, and each of them engage with people located in their "neighbourhood" in terms of opinions. This allows to conclude that, when food waste is concerned, normative appeals are most effective when they describe group behaviours occurring in the immediate situational circumstances of target individuals, as shown for other topics by Goldstein et al. (2008). Behavioural change is mediated by perceived similarity (Festinger 1954), and a certain degree of heterogeneity ensures that some committed individuals are closer to ordinary individuals. The level of polarisation and "sectarianism" (e.g., of radical pro-environmental groups) can differ between societies. While our committed agents were identified based on declared food- 
waste behaviours in Italy, other countries can show different group sizes and inter-group dynamics.

From the research point of view, this paper innovates with respect to previous works adopting a bounded confidence framework in random pairwise encounters (Weisbuch et al. 2002; Deffuant et al. 2002; Goldstein et al. 2008) by including an "interaction threshold" - agents with distant opinions are unlikely to continue to interact with, and be influenced by, each other. Future extensions might include a counterreaction, i.e. a movement in the opposite direction when others' opinions are too distant from one's prior. We have also introduced a novel "cognitive dissonance" threshold to account for the gap between stated opinions and private behaviours.

Our results allow to draw recommendations for the design of more effective policy tackling consumer food waste and associated economic and environmental costs. We recommend interventions that present food waste reduction and prevention as the social norm. Since many factors hinder people self-regulatory capacity when food is concerned, voluntary "small step" approaches based on nudges should complement policy strategies relying on regulation or market incentives (Roberto et al. 2014). The literature on recycling suggests that economic measures penalising private food waste and making it visible could incentivise the reduction of household waste over time. For instance, some Swiss municipalities that have been charging waste disposal costs per bag have seen a reduction of private waste by $40 \%$ (Carattini et al. 2018).

Based on our simulations, and as already found by Pineda and Buendía (2015) for mass media, it is recommendable for interventions nudging consumers towards less wasteful behaviours to avoid being too insistent or frequent. Indeed, a limited number of contacts seems to be enough to induce change; in turn, too insistent contacts could activate people's "confirmation bias", or increase their tolerance towards "cognitive dissonance", as they would be forced to sustain in public an opinion they do not abide by in private (Vetter 2016).

Experimental studies found that communication campaigns targeting environmental behavioural change should communicate preferably clear, achievable and easy-to-achieve messages (Vetter 2016). Based on these and on our findings, we recommend communication campaigns to be driven by practical goals, and adapted to the priors of their (potentially different) target groups without giving the impression of pursuing uncompromising principles. This is also in line with Goldstein et al. (2008), who found the use of descriptive norms more effective than traditional appeals focused solely on environmental protection. Communicating the potential for reducing household expenses thanks to better food management is expected to yield a positive impact (Priefer et al. 2016; Schanes et al. 2018; Zamri et al. 2020). Instead, targeting only "extreme" wasters or individuals who share similar (virtuous) opinions are ineffective strategies.

Favouring the opening up of groups of committed individuals to people located in their neighbourhood in terms of opinions is also key. Another type of intervention we recommend is thus to create enabling conditions for different groups - age and environmental behaviour-wise - to exchange opinions on food management, including food waste. Community centres, charitable and faith-based organisations are examples of contexts where people of various backgrounds and demographics meet and share experiences. Initiatives and events held at such organisations could trigger intergenerational (and intergroup) influence and favour the active redistribution and reuse of food that is likely to go to waste (Mousa and Freeland-Graves 2017).

The spaces for encounters should be - with priority - physical spaces, due to the higher potential of face-to-face interactions to drive behavioural change. Events and training activities focused on better household food management could be organised in shopping centres, supermarkets, and local markets. While less food waste at home could result in reduced food purchases, retail com- 
panies could benefit from such initiatives through improved trust from their clients - particularly small shops and markets, where personalised relationships are the norm - and better reputation among environmentally-concerned consumers. School-based information and training focused on food waste are also likely to initiateboth intergenerational and intergroup influence, as students from different households interact, exchange opinions, and take the new ideas they are exposed to back to their households (Ballantyne et al. 2001; Vaughan et al. 2003; Duvall and Zint 2007, Damerell et al. 2013; Marchini and Macdonald 2020).

As exemplified by the simulation case studies presented, the focus of our analysis was toward understanding the qualitative directions of food waste production under different conditions concerning the structure of social interactions - conditions that can be addressed through policy measures. For this reason, we did not aim to produce quantitative predictions, but rather study the sign of the changes. From the methodological point of view, ABMs are well suited to explore social systems building macro-outcomes from the bottom-up, and identifying how different microlevel assumptions influence the macro-outcomes (Schelling 2006). The food waste behaviour of individuals is arguably a complex system, whose determinants are many and only partially understood. Accordingly, we use our model to assess the direction and relative effectiveness of different variations to the status quo. While the structural dynamics uncovered remain valid, and could possibly be extended to other country settings, the results in terms of food waste levels should thus be considered carefully. Indeed, calibration was implemented using questionnaire data from Italy, and beside country specificities, the literature has shown that people tend to misestimate (usually underestimate) their food waste (Giordano et al. 2018). For the same reason, the values of the threshold of "cognitive dissonance" (gap between actions and opinions) could also be biased. These calibration issues, together with the requirement of data on socio-psychological constructs which are currently unavailable, represent main limitations of our model. Another limitation relates to the simplifying assumptions that we have made to make a complex phenomenon more tractable.

Rather than challenging the theoretical background underpinning the model, these caveats call for taking additional steps to better ground it into real-world situations. First, consumer food waste could be measured using more reliable methods, such as diaries or waste sorting analysis, and the resulting data used to simulate the impact of policy interventions. For example, one-to-many interactions like informational campaigns via TV or on online platforms could be simulated, as done in previous opinion dynamic studies (Pineda and Buendía 2015. Second, competing motivations related to food could be introduced, e.g. a desire to achieve a status through food abundance (Piras et al. 2021). These motivations could interact through a utility function, creating trade-offs, and also be influenced by social network. Third, efforts are required to identify the structure of social interactions specific of the setting considered. Future studies could test the impact of alternative network structures, such as the Barabási-Albert scale-free network topology (Barabási and Albert 1999), reproducing key features of human interaction networks in large-scale populations. Calibration could be implemented based on different dychotomies, e.g. between income earners and non-earners, or between social classes, given the role of income in consumer food waste generation (Setti et al. 2016). Finally, intra-household dynamics could be introduced by considering both intra-household networks and external networks. This would require information about food waste opinions of each member, while the food waste outcome would be the same for the entire household, possibly obtained through an averaging mechanism. Our ABM represents a valuable baseline where to build such additional dynamics. 


\section{Acknowledgements}

This research received funding from the Horizon 2020 Framework Programme of the European Union (project REFRESH "Resource Efficient Food and dRink for the Entire Supply cHain", Grant Agreement 641933) and the Scottish Government's Rural and Environment Science and Analytical Services Division (RESAS RD3.2.4 "Food Culture and Dietary Choice"). The views reflected in this paper represent the professional views of the authors and do not necessarily reflect the views of the funders.

\section{Appendix}
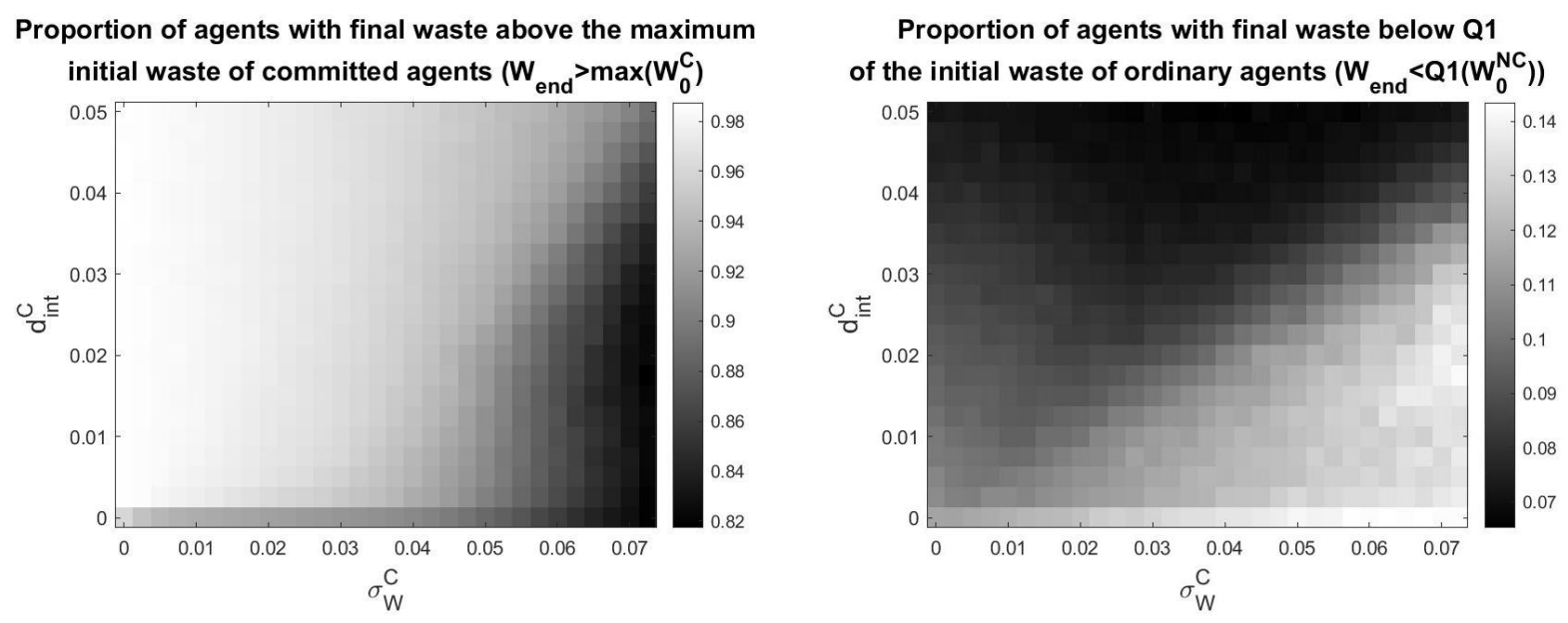

Figure 4: Additional analyses on the results of Fig. 3. Left panel: Proportion of agents with $W_{\text {end }}>\max \left(W_{0}^{C}\right)$. Right panel: Proportion of agents with $W_{\text {end }}<Q_{1}\left(W_{0}^{\text {Ord }}\right)$. 

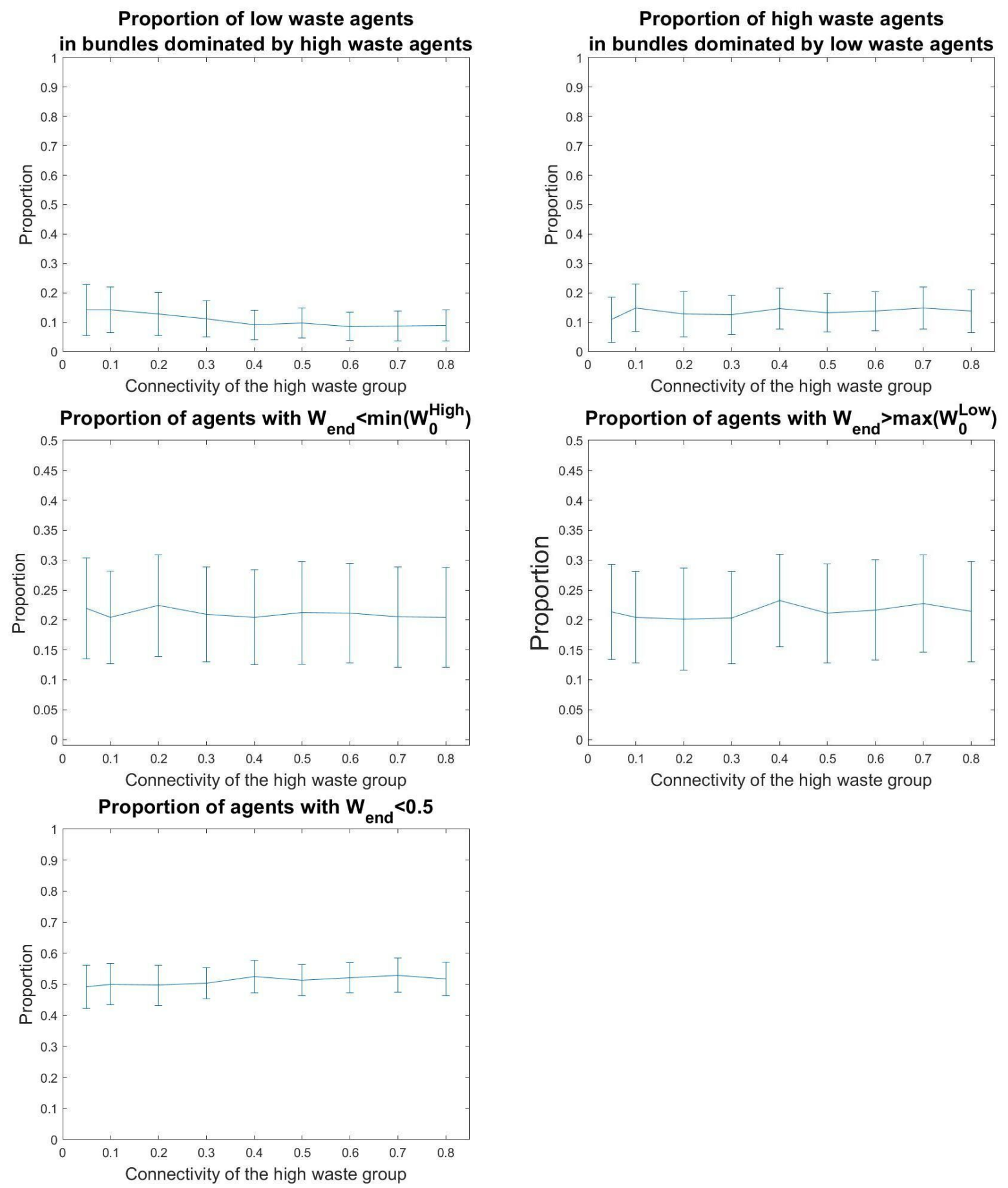

Figure 5: Impact of connectivity. Robustness checks for the relative variation of the network degree $\lambda_{j}$ across groups. Consider two groups of equal sizes; the first group has a connectivity $\lambda=0.1$; the connectivity of the second group is varied. The first group's initial waste levels follow a triangular distribution $\operatorname{Tr} i[0.33 \pm 0.33]$, those of the second group a triangular distribution $\operatorname{Tr} i[0.67 \pm 0.33]$. This figure confirm the robustness of the results in the main text. 


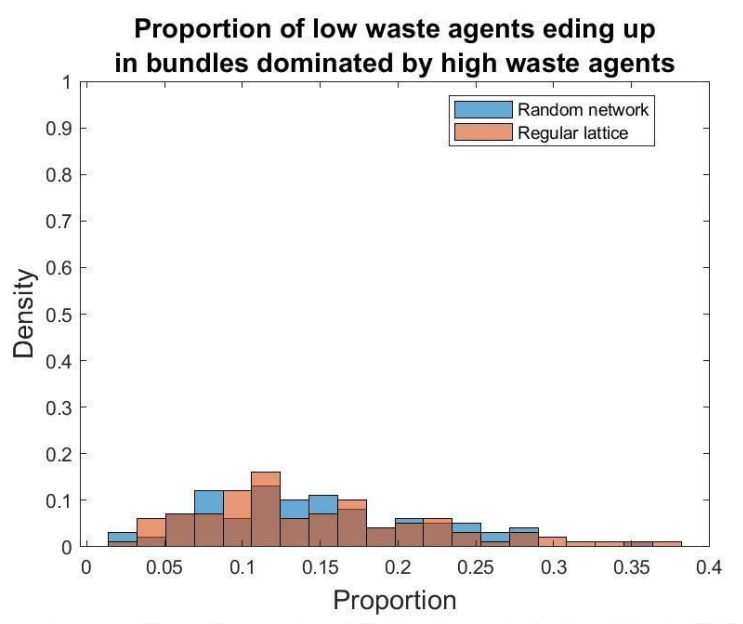

Proportion of agents with final waste below the initial minimum of high waste agents $\left(W_{\text {end }}<\min \left(W_{0}^{H i g h}\right)\right)$

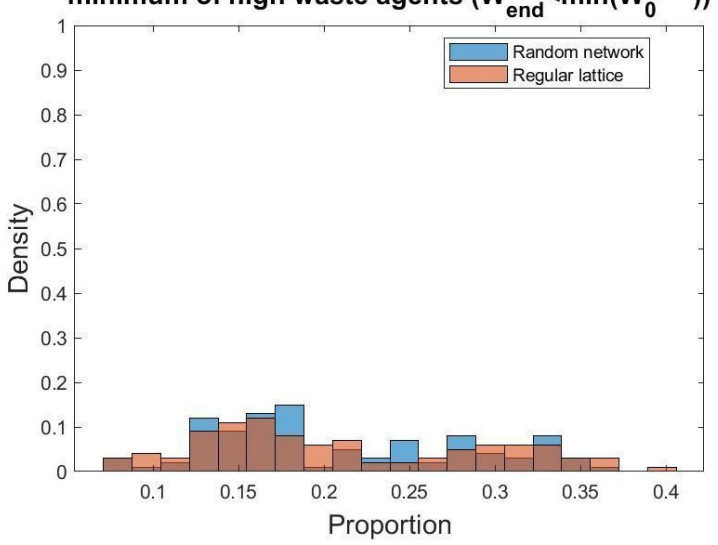

Proportion of agents with final waste below $0.5\left(\mathrm{~W}_{\text {end }}<0.5\right)$

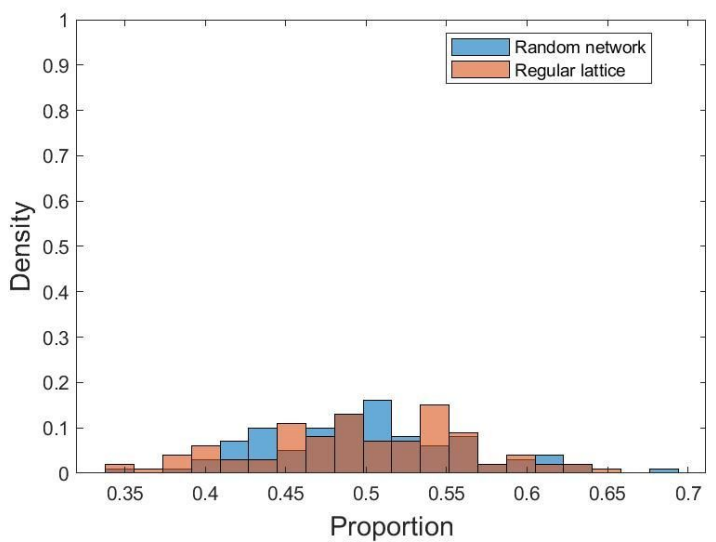

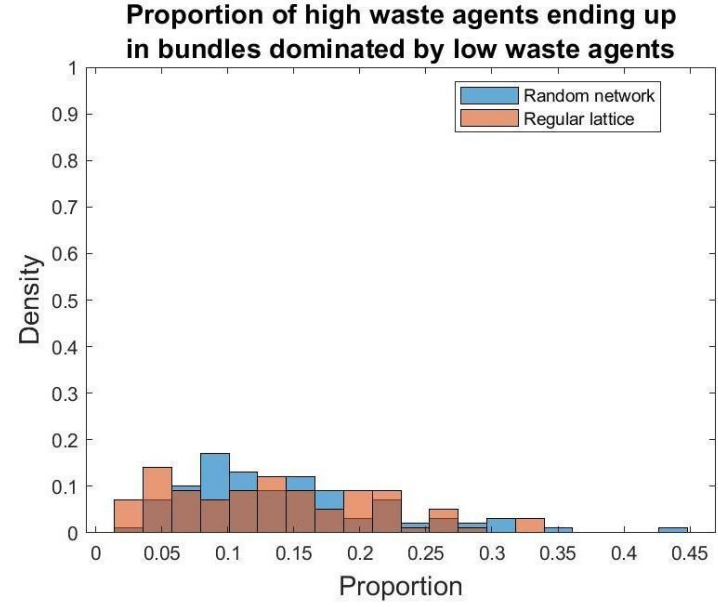

Proportion of agents with final waste above the initial

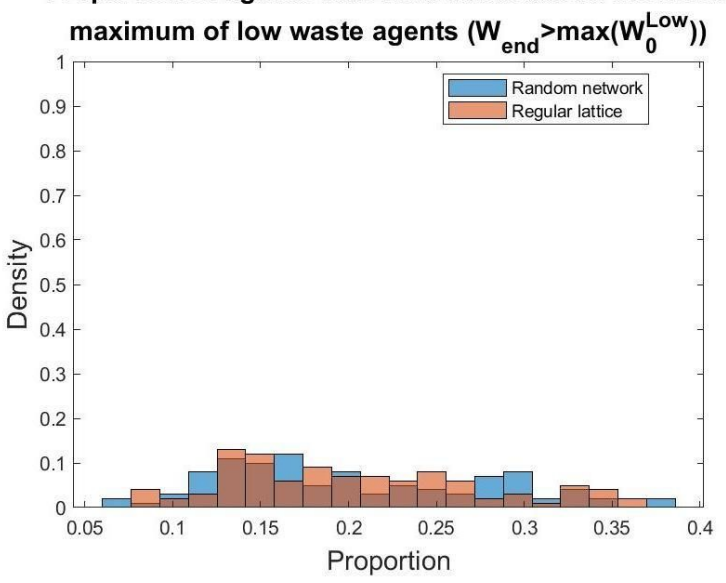

Figure 6: Lattices and Random Networks. Consider two equally-sized groups with the same degree of mixing. The first group's initial waste levels follow a triangular distribution Tri $[0.33 \pm$ $0.33]$; those of the second group a triangular distribution $\operatorname{Tr} i[0.67 \pm 0.33]$. This is the only difference between the groups. 


\section{References}

R.P. Abelson, E.E. Aronson, W.J. McGuire, T.M. Newcomb, M.J. Rosenberg, and P.H. Tannenbaum. Theories of cognitive consistency: A sourcebook. Chicago, 1968.

W. Abrahamse and L. Steg. Social influence approaches to encourage resource conservation: a meta-analysis. Global environmental change, 23(6):1773-1785, 2013.

Icek Ajzen. The theory of planned behavior. Organizational Behavior and Human Decision Processes, 50(2):179-211, 1991.

Marco Albertini. Ageing and family solidarity in Europe: patterns and driving factors of intergenerational support. The World Bank, Washington, DC, 2016.

J.J. Argo, D.W. Dahl, and R.V. Manchanda. The influence of a mere social presence in a retail context. Journal of Consumer Research, 32(2):207-212, 2012.

Roy Ballantyne, John Fien, and Jan Packer. Program effectiveness in facilitating intergenerational influence in environmental education: Lessons from the field. The journal of environmental education, 32(4):8-15, 2001.

A.L. Barabási and R Albert. Emergence of scaling in random networks. Science, 286(5439):509-512, 1999.

S Barr. Factors influencing environmental attitudes and behaviors: A UK case study of household waste management. Environment and Behavior, 39(4):435-473, 2007.

L.E. Bolton and J.W. Alba. When less is more: consumer aversion to unused utility. Journal of Consumer Psychology, 22(3):369-383, 2012.

S Bowles and H Gintis. The evolution of strong reciprocity: cooperation in heterogeneous populations. Theoretical Population Biology, 65(1):17-28, 2004.

M.G. Bublitz, L.A. Peracchio, and L.G. Block. Why did I eat that? Perspectives on food decision making and dietary restraint. Journal of Consumer Psychology, 20(3):239-258, 2010.

M Canali, P Amani, L Aramyan, M Gheoldus, G Moates, K Östergren, K Silvennoinen, K Waldron, and M Vittuari. Food waste drivers in Europe, from identification to possible interventions. Sustainability, 9(1):37, 2017.

Stefano Carattini, Andrea Baranzini, and Rafael Lalive. Is taxing waste a waste of time? evidence from a supreme court decision. Ecological Economics, 148:131-151, 2018.

E Carroni, P Pin, and S Righi. Bring a friend! privately or publicly? Management Science, 66(5): 2269-2290, 2020.

G Cecere, S Mancinelli, and M Mazzanti. Waste prevention and social preferences: The role of intrinsic and extrinsic motivations. Ecological Economics, 107:163-176, 2014. 
R.B. Cialdini, R.R. Reno, and C.A. Kallgren. A focus theory of normative conduct: recycling the concept of norms to reduce littering in public places. Journal of Personality and Social Psychology, 58(6):1015-1026, 1990.

Y Cornil, N Ordabayeva, U Kaiser, B Weber, and P Chandon. The acuity of vice: Attitude ambivalence improves visual sensitivity to increasing portion sizes. Journal of Consumer Psychology, 24 (2):177-187, 2014 .

Peter Damerell, Caroline Howe, and Eleanor J Milner-Gulland. Child-orientated environmental education influences adult knowledge and household behaviour. Environmental Research Letters, 8(1):015016, 2013.

G Deffuant, F Amblard, G Weisbuch, and T Faure. How can extremism prevail? A study based on the relative agreement interaction model. Journal of Artificial Societies and Social Simulation, $5(4), 2002$.

A Dreber, D.G. Rand, D Fudenberg, and M.A. Nowak. Winners don't punish. Nature, 452(7185): 348-351, 2008.

Jason Duvall and Michaela Zint. A review of research on the effectiveness of environmental education in promoting intergenerational learning. The Journal of Environmental Education, 38(4): 14-24, 2007.

P Erdös and A Rényi. On random graphs, I. Publicationes Mathematicae (Debrecen), 6:290-297, 1959.

D Evans. Blaming the consumer-once again: The social and material contexts of everyday food waste practices in some English households. Critical Public Health, 21(4):429-440, 2011.

E Fehr and S Gächter. Human behaviour: Egalitarian motive and altruistic punishment (reply). Nature, 433(7021):E1-E2, 2005.

L Festinger. A theory of social comparison processes. Human relations, 7(2):117-140, 1954.

Leon Festinger. Cognitive dissonance. Scientific American, 207(4):93-107, 1962.

J.H. Fowler. Altruistic punishment and the origin of cooperation. Proceedings of the National Academy of Sciences of the United States of America, 102(19):7047-7049, 2005.

J.H. Fowler, T Johnson, and O Smirnov. Egalitarian motive and altruistic punishment. Nature, 433(10.1038):137-140, 2005.

Silvia Gaiani, Sandra Caldeira, Valentina Adorno, Andrea Segrè, and Matteo Vittuari. Food wasters: Profiling consumers' attitude to waste food in italy. Waste Management, 72:17-24, 2018 .

S Galam. Sociophysics: a review of Galam models. International Journal of Modern Physics C, 19 (03):409-440, 2008.

A Galeotti. Talking, searching, and pricing. International Economic Review, 51(4):1159-1174, 2010 . 
C Giordano, S Piras, M Boschini, and L Falasconi. Are questionnaires a reliable method to measure food waste? a pilot study on italian households. British Food Journal, 2018.

N.J. Goldstein, R.B. Cialdini, and V. Griskevicius. A room with a viewpoint: Using social norms to motivate environmental conservation in hotels. Journal of Consumer Research, 35(3):472-482, 2008.

D.C. Graham-Rowe, E.and Jessop and P. Sparks. Predicting household food waste reduction using an extended theory of planned behaviour. Resources, Conservation and Recycling, 101:194-202, 2015.

E Graham-Rowe, D.C. Jessop, and P Sparks. Identifying motivations and barriers to minimising household food waste. Resources, Conservation and Recycling, 84:15-23, 2014.

Matthew James Grainger, Lusine Aramyan, Katja Logatcheva, Simone Piras, Simone Righi, Marco Setti, Matteo Vittuari, and Gavin Bruce Stewart. The use of systems models to identify food waste drivers. Global food security, 16:1-8, 2018a.

Matthew James Grainger, Lusine Aramyan, Simone Piras, Thomas Edward Quested, Simone Righi, Marco Setti, Matteo Vittuari, and Gavin Bruce Stewart. Model selection and averaging in the assessment of the drivers of household food waste to reduce the probability of false positives. PloS one, 13(2):e0192075, 2018b.

M.J. Grainger and G.B. Stewart. The jury is still out on social media as a tool for reducing food waste a response to Young et al. (2017). Resources, Conservation and Recycling, 122:407-410, 2017.

Antonio Gramsci. Quaderni dal carcere. Torino, Einaudi, 1975.

D Gunders. Wasted: How America is losing up to 40 percent of its food from farm to fork to landfill. Technical report, Natural Resources Defense Council, 2012.

J Gustavsson, C Cederberg, U Sonesson, R Van Otterdijk, and A Meybeck. Global food losses and food waste. Technical report, Food and Agriculture Organization of the United Nations, Roma, 2011.

R Hegselmann, U Krause, et al. Opinion dynamics and bounded confidence models, analysis, and simulation. Journal of Artificial Societies and Social Simulation, 5(3), 2002.

Y.E. Huh, J Vosgerau, and C.K. Morewedge. Social defaults: Observed choices become choice defaults. Journal of Consumer Research, 41(3):746-760, 2014.

M.O. Jackson. The economics of social networks. In Social Science Working Paper 1237. California Institute of Technology, 2005.

M.O. Jackson. Social and economic networks. Princeton University Press, 2010.

M.O. Jackson, B.W. Rogers, and Y. Zenou. The economic consequences of social network structure. Forthcoming, 2017. 
M.F. Ji and W Wood. Purchase and consumption habits: Not necessarily what you intend. Journal of Consumer Psychology, 17(1):261-276, 2007.

N.L. Kerr and J.M. Levine. The detection of social exclusion: Evolution and beyond. Group Dynamics: Theory, Research, and Practice, 12(1):39, 2008.

H.K. Koivupuro, H. Hartikainen, K. Silvennoinen, J.M. Katajajuuri, N. Heikintalo, A. Reinikainen, and L. Jalkanen. Influence of socio-demographical, behavioural and attitudinal factors on the amount of avoidable food waste generated in finnish households. International Journal of Consumer Studies, 36(2):183-191, 2012.

R. Kurzban and M.R. Leary. Evolutionary origins of stigmatization: The functions of social exclusion. Psychological Bulletin, 127(2):187, 2001.

C.P. Lamberton, R.W. Naylor, and K.L. Haws. Same destination, different paths: When and how does observing others' choices and reasoning alter confidence in our own choices? Journal of Consumer Psychology, 23(1):74-89, 2013.

M.K. Lapinski and R.N. Rimal. An explication of social norms. Communication Theory, 15(2): 127-147, 2005.

K Lewin. Field theory in social science. Harper, 1951.

Simonetta Longhi. Individual pro-environmental behaviour in the household context. Technical report, ISER Working Paper Series, 2013.

J Lorenz. Continuous opinion dynamics under bounded confidence: A survey. International Journal of Modern Physics C, 18(12):1819-1838, 2007.

Silvio Marchini and David W Macdonald. Can school children influence adults' behavior toward jaguars? evidence of intergenerational learning in education for conservation. Ambio, 49(4): 912-925, 2020.

B McFerran, D.W. Dahl, G.J. Fitzsimons, and A.C. Morales. I'll have what she's having: Effects of social influence and body type on the food choices of others. Journal of Consumer Research, 36(6):915-926, 2010.

H Moller, O.J. Hanssen, E Svanes, H Hartikainen, K Silvennoinen, J Gustavsson, K Östergren, F Schneider, H Soethoudt, M Canali, et al. Standard approach on quantitative techniques to be used to estimate food waste levels. Technical report, FUSIONS project, 2014a. ISBN : 82-7520-723-1 978-82-7520-723-2.

H Møller, O.J. Hanssen, J Gustavsson, K Östergren, A.A. Stenmarck, and P Dekhtyar. Report on review of (food) waste reporting methodology and practice. Technical report, FUSIONS project, 2014b. ISBN: 82-7520-713-4 978-82-7520-713-3.

Tamara Y Mousa and Jeanne H Freeland-Graves. Organizations of food redistribution and rescue. Public health, 152:117-122, 2017. 
A Mukhopadhyay, J Sengupta, and S Ramanathan. Recalling past temptations: An informationprocessing perspective on the dynamics of self-control. Journal of Consumer Research, 35(4): $586-599,2008$.

R Nishi and N Masuda. Collective opinion formation model under Bayesian updating and confirmation bias. Physical Review E, 87:062123, Jun 2013.

K Parizeau, M von Massow, and R Martin. Household-level dynamics of food waste production and related beliefs, attitudes, and behaviours in Guelph, Ontario. Waste Management, 35:207-217, 2015.

Mercedes Pineda and GM Buendía. Mass media and heterogeneous bounds of confidence in continuous opinion dynamics. Physica A: Statistical Mechanics and its Applications, 420:73-84, 2015.

Simone Piras, Francesca Pancotto, Simone Righi, Matteo Vittuari, and Marco Setti. Community social capital and status: The social dilemma of food waste. Ecological Economics, 183:106954, 2021.

Carmen Priefer, Juliane Jörissen, and Klaus-Rainer Bräutigam. Food waste prevention in europea cause-driven approach to identify the most relevant leverage points for action. Resources, Conservation and Recycling, 109:155-165, 2016.

Robert D Putnam, Robert Leonardi, and Raffaella Y. Nanetti. Making democracy work: Civic traditions in modern Italy. Princeton University Press, Princeton, NJ, 1993.

T.E. Quested, E Marsh, D Stunell, and A.D. Parry. Spaghetti soup: The complex world of food waste behaviours. Resources, Conservation and Recycling, 79:43-51, 2013.

Babak Ravandi and Nina Jovanovic. Impact of plate size on food waste: Agent-based simulation of food consumption. Resources, Conservation and Recycling, 149:550-565, 2019.

C.A. Roberto, J.L. Pomeranz, and J.O. Fisher. The need for public policies to promote healthier food consumption: A comment on Wansink and Chandon (2014). Journal of Consumer Psychology, 24(3):438-445, 2014.

Karin Schanes, Karin Dobernig, and Burcu Gözet. Food waste matters-a systematic review of household food waste practices and their policy implications. Journal of Cleaner Production, 182:978-991, 2018.

Thomas C Schelling. Micromotives and macrobehavior. WW Norton \& Company, 2006.

W.P. Schultz, A.M. Khazian, and A.C. Zaleski. Using normative social influence to promote conservation among hotel guests. Social Influence, 3(1):4-23, 2008.

Luca Secondi, Ludovica Principato, and Tiziana Laureti. Household food waste behaviour in EU-27 countries: A multilevel analysis. Food Policy, 56:25-40, 2015.

Sebastian Seebauer and Angelika Wolf. Disentangling household and individual actors in explaining private electricity consumption. Energy Efficiency, 10(1):1-20, 2017. 
J Sengupta, D.W. Dahl, and G.J. Gorn. Misrepresentation in the consumer context. Journal of Consumer Psychology, 12(2):69-79, 2002.

M. Setti, L. Falasconi, A. Segrè, I. Cusano, and M. Vittuari. Italian consumers' income and food waste behavior. British Food Journal, 118(7):1731-1746, 2016.

G. Simmel. Sociology: investigations on the forms of sociation. Duncker \& Humblot, Berlin Germany, 1908.

J.A. Simpson, V Griskevicius, and A.J. Rothman. Consumer decisions in relationships. Journal of Consumer Psychology, 22(3):304-314, 2012.

U Sonesson, F Anteson, J Davis, and P.O. Sjödén. Home transport and wastage: Environmentally relevant household activities in the life cycle of food. AMBIO: A Journal of the Human Environment, 34(4):371-375, 2005.

V Stancu, P Haugaard, and L Lähteenmäki. Determinants of consumer food waste behaviour: Two routes to food waste. Appetite, 96:7-17, 2016.

V Stefan, E van Herpen, A.A. Tudoran, and L Lähteenmäki. Avoiding food waste by Romanian consumers: The importance of planning and shopping routines. Food Quality and Preference, 28 (1):375-381, 2013.

A Stenmarck, C Jensen, T Quested, G Moates, M Buksti, B Cseh, S Juul, A Parry, A Politano, B Redlingshofer, et al. Estimates of european food waste levels. Technical report, FUSIONS project, 2016. ISBN: 978-91-88319-01-2.

P.C. Stern. New environmental theories: toward a coherent theory of environmentally significant behavior. Journal of Social Issues, 56(3):407-424, 2000.

H Tajfel and J.C. Turner. The Social Identity Theory of Intergroup Behavior. Psychology Press, 2004.

R.B. Van Baaren, R.W. Holland, K Kawakami, and Ad Van Knippenberg. Mimicry and prosocial behavior. Psychological Science, 15(1):71-74, 2004.

L.E.J. van Geffen, E. van Herpen, and J.C.M. van Trijp. Causes \& determinants of consumers food waste. project report. Technical report, EU Horizon 2020 REFRESH, 2016. ISBN: 82-7520-713-4 978-82-7520-713-3.

Christopher Vaughan, Julie Gack, Humberto Solorazano, and Robert Ray. The effect of environmental education on schoolchildren, their parents, and community members: A study of intergenerational and intercommunity learning. The Journal of Environmental Education, 34(3): 12-21, 2003.

B Verplanken, H Aarts, A Knippenberg, and A Moonen. Habit versus planned behaviour: A field experiment. British Journal of Social Psychology, 37(1):111-128, 1998.

Max Vetter. Possibilities, boundaries, and consequences of choice architecture: The case of green defaults and environmental attitudes. PhD thesis, 2016. 
G Wassermann and F Schneider. Edibles in household waste. In Proceedings of the Tenth International Waste Management and Landfill Symposium, Sardinia, pages 913-914, 2005.

M. Watson and A. Meah. Food, waste and safety: Negotiating conflicting social anxieties into the practices of domestic provisioning. The Sociological Review, 60(S2):102-120, 2013.

D.J. Watts and P.S. Dodds. Influentials, networks, and public opinion formation. Journal of Consumer Research, 34(4):441-458, 2007.

G Weisbuch, G Deffuant, F Amblard, and J.P. Nadal. Meet, discuss, and segregate! Complexity, $7(3): 55-63,2002$.

R.W. Wenlock, D.H. Buss, B.J. Derry, and E.J. Dixon. Household food wastage in Britain. British Journal of Nutrition, 43(01):53-70, 1980.

W Wood and T Hayes. Social influence on consumer decisions: Motives, modes, and consequences. Journal of Consumer Psychology, 22(3):324-328, 2012.

WRAP. Household food and drink waste: A people focus. Banbury, 2014.

C.W. Young, S Russell, and R Barkemeyer. Social media is not the 'silver bullet' to reducing household food waste, a response to grainger and stewart (2017). Resources, Conservation and Recycling, 2017a.

W Young, S.V. Russell, C.A. Robinson, and R Barkemeyer. Can social media be a tool for reducing consumers' food waste? A behaviour change experiment by a UK retailer. Resources, Conservation and Recycling, 117:195-203, 2017b.

Gesyeana Bazlyn Zamri, Nur Khaiyum Abizal Azizal, Shohei Nakamura, Koji Okada, Norul Hajar Nordin, NorÁzizi Othman, Fazrena Nadia MD Akhir, Azrina Sobian, Naoko Kaida, and Hirofumi Hara. Delivery, impact and approach of household food waste reduction campaigns. Journal of Cleaner Production, 246:118969, 2020.

M.R. Zemborain and G.V. Johar. Attitudinal ambivalence and openness to persuasion: A framework for interpersonal influence. Journal of Consumer Research, 33(4):506-514, 2007. 